\title{
Article \\ Whose Policy Uncertainty Matters in the Trade between Korea and the U.S.?
}

\author{
Mohsen Bahmani-Oskooee ${ }^{1, *(\mathbb{D})}$ and Jungho Baek ${ }^{2}$ (D) \\ 1 Department of Economics and The Center for Research on International Economics, \\ The University of Wisconsin-Milwaukee, Milwaukee, WI 53201, USA \\ 2 Department of Economics, School of Management, University of Alaska Fairbanks, \\ Fairbanks, AK 99775, USA; jbaek3@alaska.edu \\ * Correspondence: bahmani@uwm.edu
}

check for updates

Citation: Bahmani-Oskooee,

Mohsen, and Jungho Baek. 2021. Whose Policy Uncertainty Matters in the Trade between Korea and the U.S.? Journal of Risk and Financial Management 14: 520. https:// doi.org/10.3390/jrfm14110520

Academic Editor: Maria Cipollina

Received: 19 August 2021

Accepted: 20 October 2021

Published: 1 November 2021

Publisher's Note: MDPI stays neutral with regard to jurisdictional claims in published maps and institutional affiliations.

Copyright: (c) 2021 by the authors. Licensee MDPI, Basel, Switzerland. This article is an open access article distributed under the terms and conditions of the Creative Commons Attribution (CC BY) license (https:/ / creativecommons.org/licenses/by/ $4.0 /)$.

\begin{abstract}
Since the introduction of the news-based policy uncertainty measure, a few studies have looked at its impact on trade flows by using panel models and aggregate trade data. In this paper we consider the short-run and long-run response of 61 2-digit U.S. exporting industries to Korea and 49 2-digit Korean exporting industries to the U.S. to policy uncertainty measures of the U.S. and Korea. We find that both measures have short-run effects on exports of almost one-third of industries in either direction. In the long run, however, while nine U.S. exporting industries (with a trade share of $9 \%$ ) are negatively affected by the Korean uncertainty measure, only five industries (with 6\% export share) are affected by the U.S. uncertainty measure. As for the Korean exporting industries, we find that three industries with a $31 \%$ export share are affected positively by the Korean uncertainty measure and six industries with a $7 \%$ export share are affected positively by the U.S. uncertainty measure.
\end{abstract}

Keywords: economic policy uncertainty; the U.S.; Korea; industry trade

JEL Classification: F14; F40; F50

\section{Introduction}

When the international monetary system shifted from fixed to relatively more flexible exchange rates in March 1973, a new area in international economics began emerging where researchers became interested in the impact of exchange rate uncertainty or volatility on trade flows among countries. Bahmani-Oskooee and Hegerty (2007) is the latest review article on the topic. However, exchange rate uncertainty is not the only factor that may hurt trade flows. Exchange rate uncertainty or volatility could coincide with other uncertain factors that could hurt trade flows at the same time. One such measure is nothing but economic policy uncertainty, which is news-based. The measure introduced by Baker et al. (2016) captures the effects of all uncertain factors. In every country for which the measure is constructed, they search highly circulated news associated with policy uncertainty issues. Some examples of the terms searched for are: "policy", "tax", "spending", "regulation", "central bank", "budget", "deficit", "trade", "terrorism", etc. The terms such as "uncertain" or "uncertainty" are included in all searches. From the volume of the terms collected, a normalized index of uncertainty known as "Policy Uncertainty" is constructed. To learn about the path of this measure for the U.S. and Korea, we plot them in Figure 1. The spark towards the end of our study period reflects the appearance of the Covid-19 pandemic in both countries. 


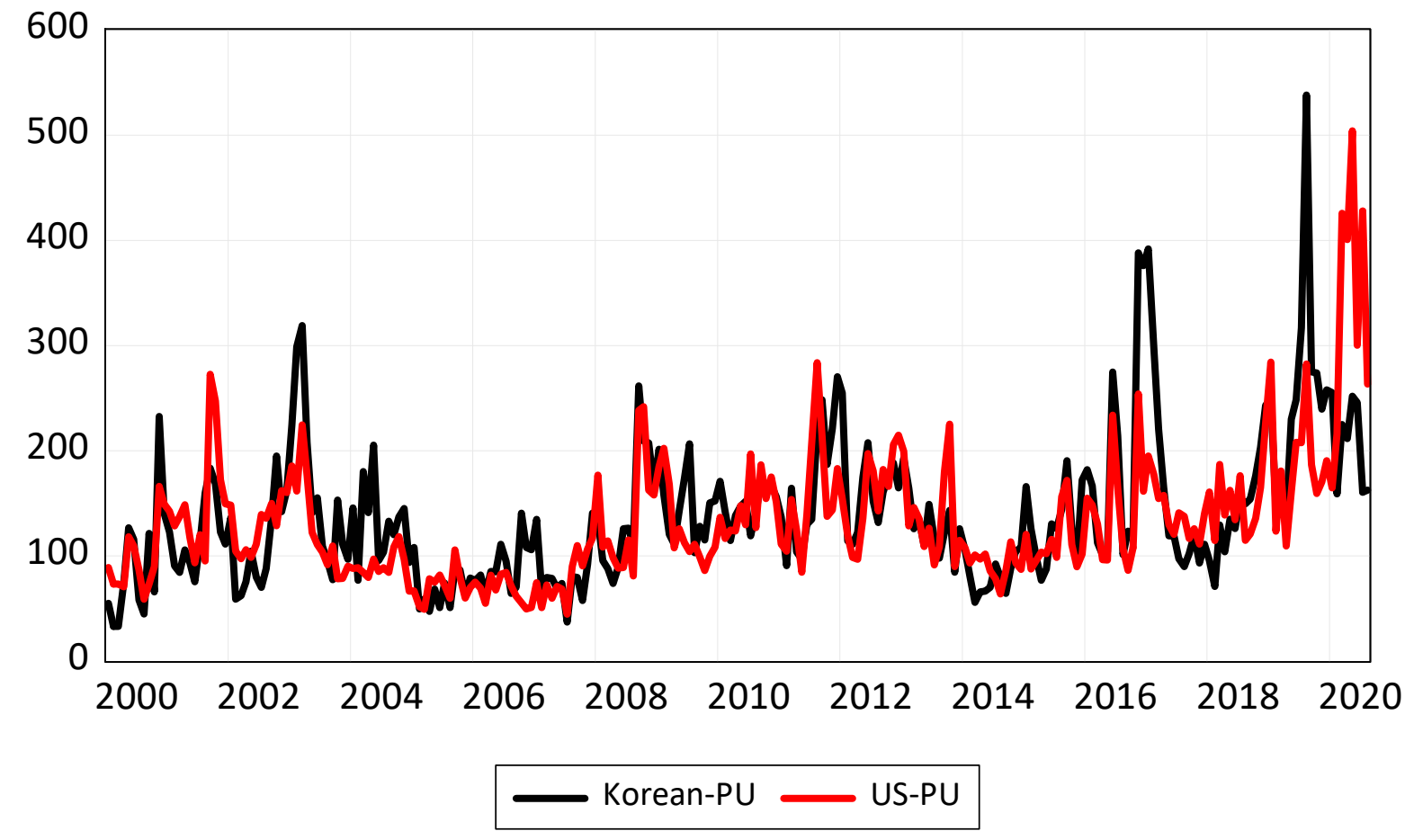

Figure 1. Monthly economic policy uncertainty indices.

Since the introduction of the policy uncertainty measure, researchers have begun to assess its impact on different macro variables. To cite some, Baker et al. (2016) themselves assessed its impact on domestic economic activity to show its adverse impact. Wang et al. (2014) assessed the response of corporate investment to the new uncertainty measure. On the other hand, Pastor and Veronesi (2013), Ko and Lee (2015), and Brogaard and Detzel (2015) investigated the response of risk premium and market returns to policy uncertainty, Kang and Ratti (2013), as well as Bahmani-Oskooee et al. (2018) and Istiak and Alam (2019), investigated the response of oil prices to the policy uncertainty measure. Furthermore, while Bahmani-Oskooee et al. (2016) assessed the impact of policy uncertainty on the demand for money in the U.S., Bahmani-Oskooee and Ghodsi (2017) considered the response of house prices in each state of the U.S., and Bahmani-Oskooee and Hasanzade (2021) looked at its impact on income distribution in each state in the U.S. To see more, one could consult the review article by Al-Thaqeb and Algharabali (2019). ${ }^{1}$

As far as the response of trade flows to the policy uncertainty measure is concerned, the literature is in its infancy and includes only Armelius et al. (2014), Han et al. (2016), and Tam (2018). Armelius et al. (2014) showed that U.S. economic policy uncertainty hurts aggregate global trade. Similarly, using a panel model that included trade data from 31 countries over the period 1999-2012, Han et al. (2016) discovered adverse effects of U.S. economic policy uncertainty. Finally, Tam (2018) employed a global vector autoregressive (GVAR) trade model, which is a panel model that included a total of 45 countries. By relying upon impulse response functions, the author finds that US policy uncertainty affects global trade flows significantly and strongly, largely due to its indirect trade linkages with the rest of the world. By contrast, China's economic policy uncertainty shock exhibits statistically significant impacts on exports and imports of its own as well as other economies, although not as pronounced as those arising from US economic policy shock.

Our main purpose in this paper is to consider trade flows between Korea and the U.S. and to try to determine whose policy uncertainty affects their trade flows more. To reduce aggregation bias embodied in the above studies, we adhere to time-series data between Korea and the U.S. To reduce the aggregation bias further, we use data at the industry level. Monthly data from 61 2-digit U.S. exporting industries to Korea and 49 2-digit Korean 
industries exporting to the U.S. over the period 2000M1-2020M7 are used to carry out the empirical exercise. The rest of the study is organized in the following manner: In Section 2 we introduce the models and estimation method, which is followed by our empirical results in Section 3. We then provide a summary in Section 4 that is followed by an Appendix A in which we provide sources of the data and definition of the variables.

\section{The Models and Methods}

A common practice in the literature to formulate a country's export and import demand model is to include a measure of economic activity and a measure of relative prices as two major determinants of a trade flow model. Indeed, such models are used to estimate the so-called Marshall-Lerner Condition. ${ }^{2}$ As such we borrow those models and after adding the two policy uncertainty measures, we begin with the following specifications:

$$
\begin{aligned}
& L n X_{i, t}^{U S}=\alpha+\alpha_{1} \operatorname{Ln} Y_{t}^{K O R}+\alpha_{2} \operatorname{LnREX} X_{t}+\alpha_{3} \operatorname{LnP} U_{t}^{U S}+\alpha_{4} \operatorname{LnP} U_{t}^{K O R}+\varepsilon_{t} \\
& L n M_{i, t}^{U S}=\beta+\beta_{1} \operatorname{Ln} Y_{t}^{U S}+\beta_{2} \operatorname{LnREX} X_{t}+\beta_{3} \operatorname{Ln} P U_{t}^{U S}+\beta_{4} \operatorname{LnP} U_{t}^{K O R}+\mu_{t}
\end{aligned}
$$

Equation (1) outlines determinants of the U.S. export of commodity $i$ to Korea denoted by $X_{\mathrm{i}}^{\mathrm{US}}$. As can be seen, the level of economic activity in Korea $\left(Y^{\mathrm{KOR}}\right)$, the real won-dollar rate $(R E X)$, the U.S. economic policy uncertainty $\left(P U^{U S}\right)$, and the Korean economic policy uncertainty $\left(P U^{\mathrm{KOR}}\right)$ are included to be the main determinants of the U.S. exports to Korea. As the Korean economy grows, we expect Korea to import more from the U.S.; hence, we expect an estimate of $\alpha_{1}$ to be positive. Finally, the real bilateral exchange rate is defined as $R E X=\left(\mathrm{NEX}^{*} \mathrm{CPI}{ }^{\mathrm{US}}\right) / \mathrm{CPI}^{\mathrm{KOR}}$ where $N E X$ is the nominal exchange rate defined as the number of won per US dollar, $\mathrm{CPI}^{\mathrm{US}}$ is the price level in the United States and $\mathrm{CPI}^{\mathrm{KOR}}$ is the price level in Korea. Thus, a decline in REX reflects a real depreciation of the US dollar. If dollar depreciation is to boost the U.S. exports to Korea, we expect an estimate of $\alpha_{2}$ to be negative. Finally, if an increase in either measure of policy uncertainty is to hurt the U.S. export, estimates of $\alpha_{3}$ and $\alpha_{4}$ are expected to be negative.

Following the above logic and reasoning, we identify determinants of the U.S. import of commodity $i$ from Korea, $M_{\mathrm{i}}^{\mathrm{US}}$, (or Korean export of commodity $i$ to the U.S.) in Equation (2). These determinants are the level of economic activity in the U.S. (YUS), the $R E X$, and two policy uncertainty measures. As the U.S. economy grows, since it imports more from Korea, an estimate of $\beta_{1}$ is expected to be positive, and if dollar depreciation is to reduce U.S. imports of commodity ii from Korea, an estimate of $\beta_{2}$ is expected to be positive. Finally, if either uncertainty measure is to hurt Korean exports to the U.S., estimates of $\beta_{3}$ and $\beta_{4}$ are expected to be negative.

The next step in our modeling approach is to convert (1) and (2) to an error-correction format so that we can also estimate the short-run effects of all exogenous variables on the dependent variables. Pesaran et al.'s (2001) ARDL bounds testing approach has a unique advantage of estimating the short-run and long-run effects in one step. We follow their approach and rely upon the following error-correction models:

$$
\begin{aligned}
& \Delta L n X_{i, t}^{U S}=a_{1}+\sum_{j=1}^{n 1} a_{2 j} \Delta L n X_{i, t-j}^{U S}+\sum_{j=0}^{n 2} a_{3 j} \Delta L n Y_{t-j}^{K O R}+\sum_{j=0}^{n 3} a_{4 j} \Delta L n R E X_{t-j}+ \\
& \sum_{j=0}^{n 4} a_{5 j} \Delta L n P U_{t-j}^{U S}+\sum_{j=0}^{n 5} a_{6 j} \Delta L n P U_{t-j}^{K O R}+\theta_{0} \operatorname{Ln} X_{i, t-1}^{U S}+\theta_{1} \operatorname{Ln} Y_{t-1}^{K O R}+ \\
& \theta_{2} \operatorname{LnREX}_{t-1}+\theta_{3} \operatorname{LnPU} U_{t-1}^{U S}+\theta_{4} \operatorname{LnPU}_{t-1}^{K O R}+\psi_{t} \\
& \Delta L n M_{i, t}^{U S}=b_{1}+\sum_{j=1}^{n 5} b_{2 j} \Delta L n M_{i, t-j}^{U S}+\sum_{j=0}^{n 6} b_{3 j} \Delta L n Y_{t-j}^{U S}+\sum_{j=0}^{n 7} b_{4 j} \Delta L n R E X_{t-j}+ \\
& \sum_{j=0}^{n 8} b_{5 j} \Delta L n P U_{t-j}^{U S}+\sum_{j=0}^{n 9} b_{6 j} \Delta L n P U_{t-j}^{K O R}+\rho_{0} L n M_{i, t-1}^{U S}+\rho_{1} \operatorname{Ln} Y_{t-1}^{U S}+ \\
& \rho_{2} \operatorname{LnREX} X_{t-1}+\rho_{3} \operatorname{LnPU} U_{t-1}^{U S}+\rho_{4} \operatorname{LnPU} U_{t-1}^{K O R}+\phi_{t}
\end{aligned}
$$

Once (3) and (4) are estimated by the OLS technique, short-run effects of any of the exogenous variables are judged by the estimates of coefficients attached to first-differenced variables, and long-run effects are inferred by the estimates of $\theta_{1}-\theta_{4}$ normalized on $-\theta_{0}$ in (3) and estimates of $\rho_{1}-\rho_{4}$ normalized on $-\rho_{0}$ in (4). To validate the long-run estimates, 
Pesaran et al. (2001) introduce two cointegration tests. The $F$ test is proposed to establish the joint significance of lagged level variables and the $t$-test is suggested to establish the significance of $\theta_{0}$ in (3) and $\rho_{0}$ in (4). ${ }^{3}$ Pesaran et al. (2001) demonstrate that the distribution of both tests is non-standard; hence, they tabulate new critical values for both tests. Since the critical values account for integrating properties of the variables, there is no need for pre-unit-root testing and variables could be a combination of $\mathrm{I}(0)$ and $\mathrm{I}(1)$, which are the properties of the majority of macro variables, and this is another advantage of this approach. ${ }^{4}$

\section{Empirical Results}

In this section, we estimate model (3) for each of the 61 2-digit U.S. exporting industries to Korea and model (4) for each of the 49 2-digit Korean exporting industries to the U.S. by using monthly data over the period 2000M1-2020M7. A maximum of eight lags are imposed on each first-differenced variable and Akaike's information criterion (AIC) is used to select an optimum specification in each case. Since the study period includes the global financial crisis of 2008, a dummy variable is included to account for it. Industries in which the dummy is significant are identified in the tables. Additionally, we have collected all required critical values in the notes to each table and used them to identify significant estimates and diagnostic statistics. We first report estimates of the U.S. export demand model to Korea in Tables 1-4. 
Table 1. Short-run coefficient estimates of Korean economic uncertainty on U.S. exports to Korea.

\begin{tabular}{|c|c|c|c|c|c|c|c|c|}
\hline \multirow{2}{*}{ Code } & \multicolumn{8}{|c|}{ \# Lags on $\triangle P U^{K O R}$} \\
\hline & 0 & 1 & 2 & 3 & 4 & 5 & 6 & 7 \\
\hline 00 Live animals & 0.063 & & & & & & & \\
\hline 01 Meat and preparations & $-0.142 * *$ & & & & & & & \\
\hline 02 Dairy products and birds' eggs & $-0.162 * *$ & $-0.153^{* *}$ & $-0.169 * *$ & & & & & \\
\hline 03 Fish, crustacean, and molluscs, and preparations thereof & $-0.222 *$ & & & & & & & \\
\hline 04 Cereals and cereal preparations & -0.005 & & & & & & & \\
\hline 05 Vegetables and fruit & $-0.170 * *$ & -0.055 & -0.018 & 0.026 & 0.062 & $0.116^{* *}$ & $0.129 * *$ & \\
\hline 06 Sugar, sugar preparations, and honey & -0.029 & & & & & & & \\
\hline 07 Coffee, tea, cocoa, spices, and manufactures thereof & -0.015 & & & & & & & \\
\hline 08 Feeding stuff for animals (not including unmilled cereals) & 0.014 & & & & & & & \\
\hline 09 Miscellaneous edible products and preparations & -0.067 & & & & & & & \\
\hline 11 Beverages & 0.059 & $-0.188^{* *}$ & $-0.099 *$ & & & & & \\
\hline 21 Hides, skins and furskins, raw & 0.054 & & & & & & & \\
\hline 22 Oil seeds and oleaginous fruit & 0.343 & $-0.602 * *$ & & & & & & \\
\hline 23 Crude rubber (including synthetic and reclaimed) & -0.038 & $0.259 * *$ & & & & & & \\
\hline 24 Cork and wood & -0.034 & & & & & & & \\
\hline 25 Pulp and waste paper & -0.031 & $-0.060 *$ & & & & & & \\
\hline 26 Textile fibers (not wool tops) and their wastes (not in yarn) & 0.005 & & & & & & & \\
\hline 27 Crude fertilizer and crude minerals & -0.053 & & & & & & & \\
\hline 28 Metalliferous ores and metal scrap & 0.061 & & & & & & & \\
\hline 41 Animal oils and fats & -0.203 & & & & & & & \\
\hline 42 Fixed vegetable oils and fats & -0.388 & 0.341 & $0.757^{* *}$ & $0.909 * *$ & & & & \\
\hline 43 Animal and vegetable oils and fats, processed and waxes & 0.030 & & & & & & & \\
\hline 51 Organic chemicals & $0.075 *$ & -0.007 & -0.066 & -0.153 ** & $-0.076^{*}$ & $-0.102 * *$ & & \\
\hline 52 Inorganic chemicals & 0.102 & & & & & & & \\
\hline 53 Dyeing, tanning, and coloring materials & -0.033 & & & & & & & \\
\hline 54 Medicinal and pharmaceutical products & -0.070 & & & & & & & \\
\hline 55 Oils and perfume materials; toilet and cleansing preparations & -0.038 & & & & & & & \\
\hline 56 Fertilizers, manufactured & 0.119 & & & & & & & \\
\hline 57 Explosives and pyrotechnic products & -0.005 & & & & & & & \\
\hline 58 Artificial resins and plastic materials, and cellulose esters, etc & $-0.101 * *$ & & & & & & & \\
\hline 59 Chemical materials and products, nes & -0.048 & $-0.089 * *$ & -0.012 & $0.066^{*}$ & & & & \\
\hline
\end{tabular}


Table 1. Cont.

\begin{tabular}{|c|c|c|c|c|c|c|c|c|}
\hline \multirow{2}{*}{ Code } & \multicolumn{8}{|c|}{ \# Lags on $\triangle P U^{K O R}$} \\
\hline & 0 & 1 & 2 & 3 & 4 & 5 & 6 & 7 \\
\hline 61 Leather, leather manufactures, nes, and dressed furskins & -0.185 * & & & & & & & \\
\hline 62 Rubber manufactures, nes \# & 0.031 & & & & & & & \\
\hline 63 Cork and wood, cork manufactures & 0.009 & & & & & & & \\
\hline 64 Paper, paperboard, and articles of pulp, of paper, or of paperboard & -0.051 * & & & & & & & \\
\hline 65 Textile yarn, fabrics, made-up articles, nes, and related products & -0.036 & & & & & & & \\
\hline 66 Non-metallic mineral manufactures, nes & 0.014 & & & & & & & \\
\hline 67 Iron and steel & 0.069 & $-0.113 *$ & & & & & & \\
\hline 68 Non-ferrous metals. & -0.025 & & & & & & & \\
\hline 71 Power generating machinery and equipment & -0.087 & & & & & & & \\
\hline 72 Machinery specialized for particular industries & -0.068 & & & & & & & \\
\hline 73 Metalworking machinery & 0.009 & & & & & & & \\
\hline 74 General industrial machinery and equipment, nes, and parts of, nes & -0.049 & & & & & & & \\
\hline 75 Office machines and automatic data processing equipment & 0.008 & & & & & & & \\
\hline 76 Telecommunications, sound recording, and reproducing equipment & -0.055 & -0.168 ** & $-0.265^{* *}$ & -0.062 & 0.015 & 0.103 & $0.227^{* *}$ & $0.157^{* *}$ \\
\hline 77 Electric machinery, apparatus and appliances, nes, and parts, nes & 0.012 & & & & & & & \\
\hline 78 Road vehicles & -0.010 & & & & & & & \\
\hline 79 Other transport equipment & -0.045 & $0.415 * *$ & & & & & & \\
\hline 81 Sanitary, plumbing, heating, lighting fixtures, and fittings, nes & -0.041 & & & & & & & \\
\hline 82 Furniture and parts thereof & 0.042 & 0.048 & 0.016 & 0.093 & 0.195 ** & $0.155 * *$ & $0.132 * *$ & $0.104^{* *}$ \\
\hline 84 Articles of apparel and clothing accessories & -0.037 & -0.108 & $-0.125 *$ & $-0.275^{* *}$ & $-0.191 * *$ & 0.069 & $-0.139 * *$ & \\
\hline 85 Footwear & 0.086 & & & & & & & \\
\hline 87 Professional, scientific, controlling instruments, apparatus, nes & -0.064 * & $-0.101 * *$ & $-0.096 * *$ & -0.048 & -0.026 & 0.007 & $0.064 * *$ & $0.069 * *$ \\
\hline 88 Photographic equipment and supplies, optical goods, watches, etc & -0.014 & 0.012 & $-0.083 * *$ & & & & & \\
\hline 89 Miscellaneous manufactured articles, nes & -0.068 & & & & & & & \\
\hline 93 Special transactions, commodity not classified according to class & $-0.158^{* *}$ & & & & & & & \\
\hline 99 Estimate of non-Canadian low-value shipments & 0.009 & & & & & & & \\
\hline
\end{tabular}

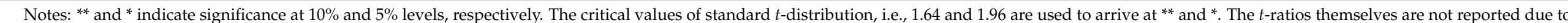
space constraints but they are available upon request. nes = not elsewhere specified. \# next to an industry's name indicates that the global financial crisis dummy was significant. 
Table 2. Short-Run coefficient estimates of US economic policy uncertainty on U.S. exports to Korea.

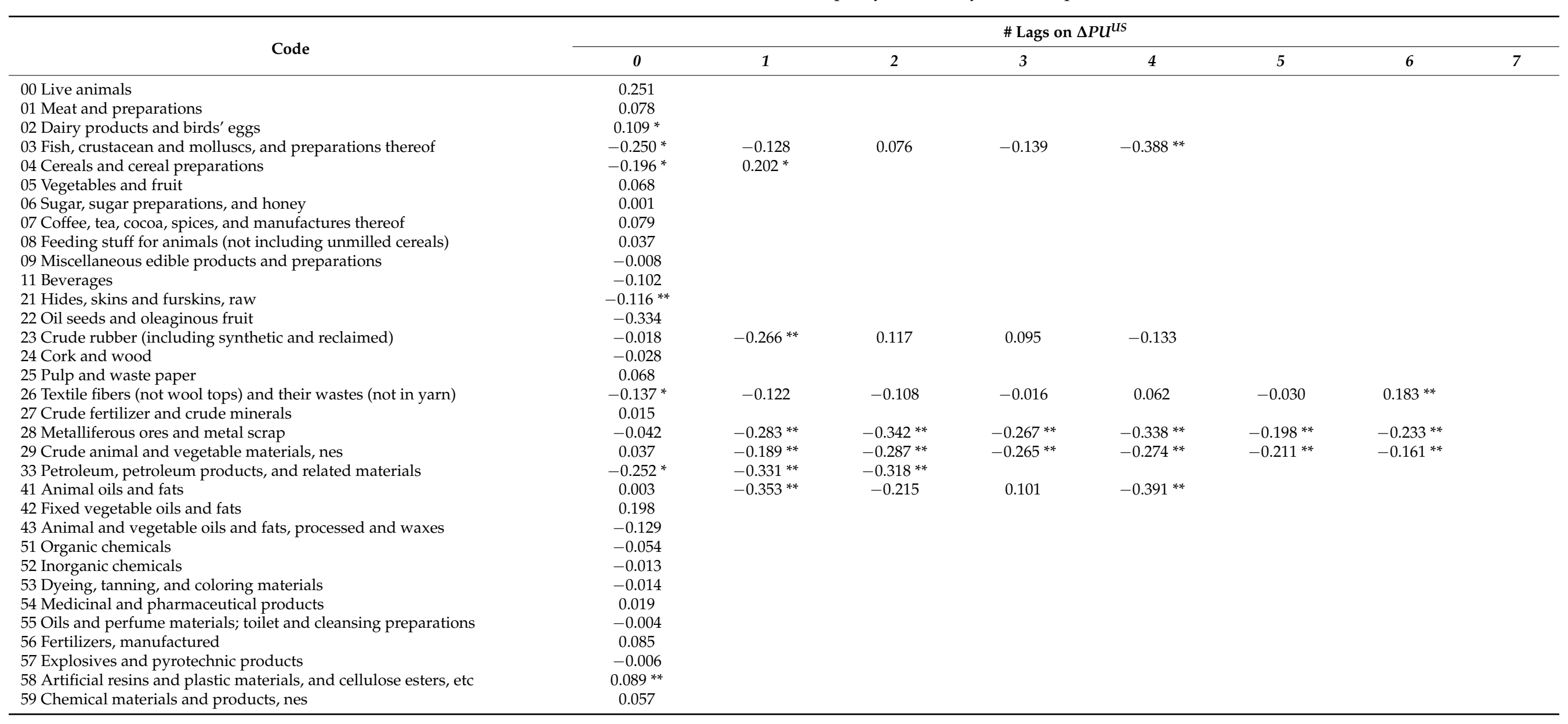


Table 2. Cont.

\begin{tabular}{|c|c|c|c|c|c|c|c|c|}
\hline \multirow{2}{*}{ Code } & \multicolumn{8}{|c|}{ \# Lags on $\triangle P U^{U S}$} \\
\hline & 0 & 1 & 2 & 3 & 4 & 5 & 6 & 7 \\
\hline 61 Leather, leather manufactures, nes, and dressed furskins & 0.092 & & & & & & & \\
\hline 62 Rubber manufactures, nes \# & -0.087 & & & & & & & \\
\hline 63 Cork and wood, cork manufactures & $-0.200 * *$ & -0.094 & $-0.176^{* *}$ & -0.083 & -0.096 & 0.128 & -0.004 & 0.163 ** \\
\hline 64 Paper, paperboard, and articles of pulp, of paper, or of paperboard & 0.019 & & & & & & & \\
\hline 65 Textile yarn, fabrics, made-up articles, nes, and related products & -0.015 & & & & & & & \\
\hline 66 Non-metallic mineral manufactures, nes & -0.016 & & & & & & & \\
\hline 67 Iron and steel & $-0.131 *$ & 0.049 & $-0.141^{* *}$ & & & & & \\
\hline 68 Non-ferrous metals. & -0.087 & & & & & & & \\
\hline 71 Power generating machinery and equipment & $0.131 *$ & 0.079 & 0.107 & $0.211 * *$ & & & & \\
\hline 72 Machinery specialized for particular industries & 0.033 & & & & & & & \\
\hline 73 Metalworking machinery & -0.103 & $0.209 * *$ & & & & & & \\
\hline 74 General industrial machinery and equipment, nes, and parts of, nes & -0.023 & & & & & & & \\
\hline 75 Office machines and automatic data processing equipment & -0.071 & & & & & & & \\
\hline 76 Telecommunications, sound recording, and reproducing equipment & -0.026 & $0.299 * *$ & $0.287^{* *}$ & $0.182 * *$ & $0.228 * *$ & 0.033 & & \\
\hline 77 Electric machinery, apparatus and appliances, nes, and parts, nes & -0.033 & & & & & & & \\
\hline 78 Road vehicles & -0.073 & $-0.116^{*}$ & & & & & & \\
\hline 79 Other transport equipment & 0.040 & $-0.474^{* *}$ & & & & & & \\
\hline 81 Sanitary, plumbing, heating, lighting fixtures, and fittings, nes & -0.170 & & & & & & & \\
\hline 82 Furniture and parts thereof & 0.009 & & & & & & & \\
\hline 84 Articles of apparel and clothing accessories & -0.030 & 0.027 & $0.250 * *$ & $0.233 * *$ & $0.194^{* *}$ & 0.071 & $0.194^{* *}$ & \\
\hline 85 Footwear & -0.115 & & & & & & & \\
\hline 87 Professional, scientific, controlling instruments, apparatus, nes & 0.067 & $0.137^{* *}$ & $0.189 * *$ & $0.149^{* *}$ & & & & \\
\hline 88 Photographic equipment and supplies, optical goods, watches, etc & 0.017 & & & & & & & \\
\hline 89 Miscellaneous manufactured articles, nes & -0.007 & & & & & & & \\
\hline 93 Special transactions, commodity not classified according to class & 0.039 & & & & & & & \\
\hline 99 Estimate of non-Canadian low-value shipments & -0.003 & & & & & & & \\
\hline
\end{tabular}

Notes: ** and * indicate significance at $10 \%$ and $5 \%$ levels, respectively. The critical values of standard $t$-distribution, i.e., 1.64 and 1.96 are used to arrive
space constraints but they are available upon request. \# next to an industry's name indicates that the global financial crisis dummy was significant. 
Table 3. Long-run coefficient estimates of U.S. export models.

\begin{tabular}{|c|c|c|c|c|c|c|}
\hline Industries & Share $^{\mathrm{c}}$ & Constant & $\operatorname{Ln} Y_{t}{ }^{K O R}$ & $\operatorname{Ln} R E X_{t}$ & $\operatorname{Ln} \mathrm{PU}_{t} \mathrm{KOR}^{\mathrm{NOR}}$ & $\operatorname{Ln} \mathrm{PU}_{t}{ }^{\mathrm{US}}$ \\
\hline 00 Live animals \# ${ }^{a}$ & $0.045 \%$ & $10.571(5.291)^{* *}$ & $1.363(4.093) * *$ & $-0.743(0.807)^{\mathrm{b}}$ & $0.067(0.339)$ & $0.267(1.157)$ \\
\hline 01 Meat and preparations & $4.343 \%$ & $-0.430(3.411) * *$ & $2.197(2.120) * *$ & $1.408(0.471)$ & $-0.232(0.355)$ & $0.828(1.173)$ \\
\hline 02 Dairy products and birds' eggs \# & $0.532 \%$ & $2.283(4.651) * *$ & $2.773(6.204) * *$ & $-1.132(0.870)$ & $-0.169(0.568)$ & $0.488(1.716)$ * \\
\hline 03 Fish, crustacean and molluscs, and preparations thereof \# & $0.906 \%$ & $15.737(6.081) * *$ & $0.132(0.478)$ & $0.033(0.041)$ & $-0.238(1.591)$ & $0.111(0.488)$ \\
\hline 04 Cereals and cereal preparations \# & $1.561 \%$ & $3.565(6.644) * *$ & $1.731(3.060)^{* *}$ & $0.041(0.026)$ & $-0.423(1.065)$ & $-0.055(0.127)$ \\
\hline 05 Vegetables and fruit \# & $1.745 \%$ & $1.387(4.347) * *$ & $2.147(7.541) * *$ & $0.565(0.720)$ & $-0.385(1.787)$ * & $0.234(1.336)$ \\
\hline 06 Sugar, sugar preparations, and honey \# & $0.076 \%$ & $3.629(3.696) * *$ & $0.892(1.874) *$ & $-0.971(0.715)$ & $-0.148(0.521)$ & $0.007(0.021)$ \\
\hline 07 Coffee, tea, cocoa, spices, and manufactures thereof \# & $0.314 \%$ & $1.499(5.324) * *$ & $2.051(11.650) * *$ & $0.440(0.894)$ & $-0.029(0.266)$ & $0.153(1.253)$ \\
\hline 08 Feeding stuff for animals (not including unmilled cereals) & $1.282 \%$ & $0.629(6.418) * *$ & $2.396(11.109) * *$ & $0.657(1.098)$ & $0.028(0.219)$ & $0.075(0.514)$ \\
\hline 11 Beverages & $0.190 \%$ & $5.212(3.173)^{* *}$ & $-0.341(0.255)$ & $-5.161(1.314)$ & $1.065(1.188)$ & $0.639(0.840)$ \\
\hline 21 Hides, skins and furskins, raw & $0.266 \%$ & $-1.486(2.962) * *$ & $-1.273(0.952)$ & $-1.231(0.328)$ & $-0.974(0.929)$ & $2.096(1.099)$ \\
\hline 22 Oil seeds and oleaginous fruit \# & $0.792 \%$ & $21.274(9.667)^{* *}$ & $-0.579(1.872)^{*}$ & $0.041(0.047)$ & $0.251(1.185)$ & $0.306(1.327)$ \\
\hline 23 Crude rubber (including synthetic and reclaimed) & $0.111 \%$ & $6.626(5.215) * *$ & $0.926(2.217) * *$ & $-0.893(0.739)$ & $-0.308(1.152)$ & $0.111(0.302)$ \\
\hline 24 Cork and wood \# & $0.142 \%$ & $5.761(3.120) * *$ & $-0.437(0.875)$ & $-0.525(0.380)$ & $-0.136(0.455)$ & $-0.112(0.320)$ \\
\hline 25 Pulp and waste paper & $0.511 \%$ & $6.063(5.901) * *$ & $0.049(0.244)$ & $-0.221(0.404)$ & $-0.264(1.944)$ * & $0.209(1.561)$ \\
\hline 26 Textile fibers (not wool tops) and their wastes (not in yarn) \# & $0.385 \%$ & $5.865(4.919) * *$ & $0.240(0.682)$ & $-0.076(0.076)$ & $0.013(0.074)$ & $0.109(0.359)$ \\
\hline 27 Crude fertilizer and crude minerals \# & $0.114 \%$ & $8.664(7.621) * *$ & $-0.122(1.147)$ & $0.104(0.351)$ & $-0.096(1.531)$ & $0.027(0.366)$ \\
\hline 28 Metalliferous ores and metal scrap \# & $2.678 \%$ & $12.162(6.174) * *$ & $0.549(1.265)$ & $-2.771(2.244)^{* *}$ & $0.162(0.781)$ & $0.487(1.338)$ \\
\hline 29 Crude animal and vegetable materials, nes \# & $0.188 \%$ & $8.871(9.292) * *$ & $0.639(3.431)^{* *}$ & $-0.668(1.251)$ & $-0.233(2.285)^{* *}$ & $0.649(4.058)$ ** \\
\hline 33 Petroleum, petroleum products, and related materials \# & $17.503 \%$ & $1.632(1.293)$ & $1.589(0.376)$ & $-3.495(0.280)$ & $0.272(0.122)$ & $1.194(0.356)$ \\
\hline 42 Fixed vegetable oils and fats & $0.473 \%$ & $-6.639(4.517)^{* *}$ & $1.695(0.848)$ & $3.426(0.611)$ & $0.202(0.153)$ & $0.643(0.533)$ \\
\hline 43 Animal and vegetable oils and fats, processed and waxes & $0.012 \%$ & $5.726(4.524)^{* *}$ & $0.394(0.635)$ & $-0.338(0.196)$ & $0.077(0.207)$ & $-0.334(0.766)$ \\
\hline 51 Organic chemicals & $4.545 \%$ & $7.253(4.774) * *$ & $-0.007(0.021)$ & $-1.808(1.983)$ ** & $0.684(2.796)^{* *}$ & $-0.217(1.034)$ \\
\hline 52 Inorganic chemicals \# & $1.145 \%$ & $17.387(15.138)^{* *}$ & $0.838(6.083) * *$ & $-0.716(1.826)^{*}$ & $0.108(1.356)$ & $-0.014(0.147)$ \\
\hline 53 Dyeing, tanning, and coloring materials \# & $0.549 \%$ & $1.536(6.511) * *$ & $1.281(6.851) * *$ & $1.022(1.925)$ * & $-0.096(0.884)$ & $-0.040(0.312)$ \\
\hline 54 Medicinal and pharmaceutical products & $1.922 \%$ & $-2.337(5.281)^{* *}$ & $2.658(13.398) * *$ & $1.615(2.883) * *$ & $-0.156(1.336)$ & $0.042(0.301)$ \\
\hline 55 Oils and perfume materials; toilet and cleansing preparations \# & $1.093 \%$ & $0.811(4.018)^{* *}$ & $1.605(8.668)^{* *}$ & $1.093(2.078) * *$ & $-0.148(1.322)$ & $-0.015(0.122)$ \\
\hline 56 Fertilizers, manufactured & $0.264 \%$ & $22.819(4.497) * *$ & $-1.450(2.398) * *$ & $-2.651(1.499)$ & $0.207(0.558)$ & $0.147(0.359)$ \\
\hline 57 Explosives and pyrotechnic products \# & $1.927 \%$ & $9.900(7.572) * *$ & $0.972(12.307)^{* *}$ & $-0.668(3.057)^{* *}$ & $-0.008(0.182)$ & $-0.012(0.219)$ \\
\hline 58 Artificial resins and plastic materials, and cellulose esters, etc \# & $0.400 \%$ & $11.822(8.409) * *$ & $0.445(5.721)^{* *}$ & $-0.260(1.197)$ & $-0.141(2.933)^{* *}$ & $0.125(2.301)$ ** \\
\hline 59 Chemical materials and products, nes & $2.475 \%$ & $7.103(7.389)^{* *}$ & $1.302(10.535) * *$ & $-0.089(0.258)$ & $-0.0004(0.004)$ & $0.099(1.263)$ \\
\hline 61 Leather, leather manufactures, nes, and dressed furskins & $0.007 \%$ & $2.924(2.431) * *$ & $-1.815(0.594)$ & $-3.637(0.406)$ & $-3.581(1.093)$ & $1.782(0.667)$ \\
\hline 62 Rubber manufactures, nes \# & $0.263 \%$ & $0.077(3.811) * *$ & $2.093(4.015) * *$ & $1.052(0.749)$ & $0.196(0.647)$ & $-0.550(1.315)$ \\
\hline 63 Cork and wood, cork manufactures \# & $0.046 \%$ & $7.012(5.319) * *$ & $-0.713(1.548)$ & $-0.935(0.712)$ & $0.032(0.143)$ & $0.031(0.078)$ \\
\hline 64 Paper, paperboard, and articles of pulp, of paper, or of paperboard \# & $0.497 \%$ & $4.988(4.753) * *$ & $0.557(4.435) * *$ & $0.281(0.813)$ & $-0.129(1.585)$ & $0.048(0.566)$ \\
\hline 65 Textile yarn, fabrics, made-up articles, nes, and related products & $0.269 \%$ & $0.816(3.935)^{* *}$ & $0.633(1.851) *$ & $1.461(1.391)$ & -0.207 (1.158) & $-0.084(0.415)$ \\
\hline
\end{tabular}


Table 3. Cont.

\begin{tabular}{|c|c|c|c|c|c|c|}
\hline Industries & Share $^{\mathrm{c}}$ & Constant & $\operatorname{Ln} Y_{t}^{K O R}$ & $\operatorname{Ln} R E X_{t}$ & $\operatorname{Ln} \mathrm{PU}_{t}^{\mathrm{KOR}}$ & $\operatorname{Ln} \mathrm{PU}_{t}{ }^{\mathrm{US}}$ \\
\hline 66 Non-metallic mineral manufactures, nes \# & $0.648 \%$ & $7.629(7.263) * *$ & $0.859(7.421)^{* *}$ & $-0.467(1.404)$ & $0.030(0.461)$ & $0.099(1.183)$ \\
\hline 67 Iron and steel \# & $0.319 \%$ & $3.906(4.303) * *$ & $0.916(1.238)$ & $-2.269(1.036)$ & $1.315(1.988)^{* *}$ & $-0.691(1.254)$ \\
\hline 68 Non-ferrous metals \# & $1.178 \%$ & $9.945(4.706)^{* *}$ & $0.749(2.343) * *$ & $-2.034(2.183)^{* *}$ & $-0.071(0.409)$ & $0.119(0.529)$ \\
\hline 69 Manufactures of metals, nes & $1.000 \%$ & $2.110(5.470) * *$ & $1.575(10.064) * *$ & $0.863(2.011)^{* *}$ & $-0.405(3.310) * *$ & $0.133(1.346)$ \\
\hline 71 Power generating machinery and equipment & $1.811 \%$ & $10.889(6.889)^{* *}$ & $0.589(3.099)^{* *}$ & $-0.050(0.092)$ & $-0.135(1.402)$ & $-0.104(0.685)$ \\
\hline 72 Machinery specialized for particular industries & $6.437 \%$ & $2.289(5.321) * *$ & $1.899(5.172)^{* *}$ & $0.491(0.495)$ & $-0.228(1.102)$ & $0.113(0.457)$ \\
\hline 73 Metalworking machinery & $0.178 \%$ & $9.253(4.454)^{* *}$ & $-1.604(2.492) * *$ & $-1.557(0.826)$ & $0.038(0.107)$ & $-0.935(2.003) * *$ \\
\hline 74 General industrial machinery and equipment, nes, and parts of, nes & $2.554 \%$ & $3.319(4.233) * *$ & $0.962(4.198) * *$ & $0.515(0.799)$ & $-0.174(1.278)$ & $-0.081(0.501)$ \\
\hline 75 Office machines and automatic data processing equipment & $1.177 \%$ & $3.524(3.676) * *$ & $-0.679(0.889)$ & $0.065(0.039)$ & $0.052(0.153)$ & $-0.446(0.963)$ \\
\hline 76 Telecommunications, sound recording, and reproducing equipment & $1.042 \%$ & $4.654(4.796) * *$ & $-0.245(0.584)$ & $0.983(0.839)$ & $0.187(0.663)$ & $-0.641(1.820) *$ \\
\hline 77 Electric machinery, apparatus and appliances, nes, and parts, nes \# & $8.815 \%$ & $6.702(4.565) * *$ & $-0.693(3.915)^{* *}$ & $-1.279(2.566) * *$ & $0.056(0.565)$ & $0.013(0.106)$ \\
\hline 78 Road vehicles \# & $3.962 \%$ & $6.102(6.399) * *$ & $1.695(4.812)^{* *}$ & $-1.435(1.434)$ & $-0.033(0.172)$ & $0.155(0.586)$ \\
\hline 79 Other transport equipment & $4.564 \%$ & $4.593(5.299) * *$ & $0.932(1.958) *$ & $1.335(0.979)$ & $-0.622(1.839)$ * & $-0.017(0.045)$ \\
\hline 81 Sanitary, plumbing, heating, lighting fixtures, and fittings, nes & $0.130 \%$ & $-1.593(4.188) * *$ & $1.076(1.840) *$ & $2.704(1.688)$ * & $-0.144(0.437)$ & $-0.593(1.364)$ \\
\hline 82 Furniture and parts thereof & $0.130 \%$ & $-1.068(3.575) * *$ & $1.963(2.301)^{* *}$ & $2.694(1.133)$ & $-1.188(1.762) *$ & $0.061(0.143)$ \\
\hline 83 Travel goods, handbags, and similar containers & $0.031 \%$ & $9.379(3.416) * *$ & $-1.758(2.113) * *$ & $-3.003(1.435)$ & $0.822(1.583)$ & $-0.479(0.986)$ \\
\hline 84 Articles of apparel and clothing accessories & $0.186 \%$ & $5.342(4.639) * *$ & $1.349(4.237) * *$ & $-0.749(0.833)$ & $0.334(1.544)$ & $-0.378(1.376)$ \\
\hline 85 Footwear & $0.067 \%$ & $5.134(3.753) * *$ & $-1.691(0.353)$ & $-11.662(0.931)$ & $1.795(0.838)$ & $-2.416(0.957)$ \\
\hline 87 Professional, scientific, controlling instruments, apparatus, nes & $4.707 \%$ & $5.523(5.084) * *$ & $0.985(3.605)^{* *}$ & $-0.643(0.581)$ & $0.329(1.562)$ & $-0.574(1.951)$ * \\
\hline 88 Photographic equipment and supplies, optical goods, watches, etc \# & $0.802 \%$ & $8.179(4.641) * *$ & $-0.523(1.342)$ & $-2.599(2.416) * *$ & $0.176(0.802)$ & $0.075(0.302)$ \\
\hline 89 Miscellaneous manufactured articles, nes & $2.359 \%$ & $8.434(4.614) * *$ & $0.544(2.572)^{* *}$ & $-0.075(0.131)$ & $-0.138(1.039)$ & $-0.015(0.103)$ \\
\hline 99 Estimate of non-Canadian low-value shipments & $0.946 \%$ & $1.807(3.740)^{* *}$ & $0.675(2.467) * *$ & $0.067(0.091)$ & $0.067(0.429)$ & $-0.019(0.103)$ \\
\hline
\end{tabular}

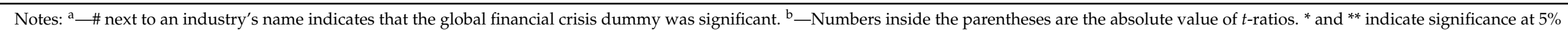

levels and $10 \%$ levels respectively. ${ }^{c}$ Share represents \% share of each industry's total U.S. exports to Korea. 
Table 4. Diagnostic statistics associated with U.S. export models.

\begin{tabular}{|c|c|c|c|c|c|c|c|}
\hline \multirow[b]{2}{*}{ Industries } & \multicolumn{7}{|c|}{ Diagnostics } \\
\hline & F Stat ${ }^{a}$ & $\hat{\theta}_{0}(t \text {-Test })^{\mathbf{b}}$ & $L M^{c}$ & $R_{E S E T}^{d}$ & cUSUM & cusumsQ & $\operatorname{Adj} . R^{2}$ \\
\hline 00 Live animals & $5.50^{* *}$ & $-0.941(5.292) * *$ & 0.129 & 0.785 & $\mathrm{~S}$ & $\mathrm{~S}$ & 0.63 \\
\hline 01 Meat and preparations & 2.34 & $-0.094(3.446)$ & 0.349 & 0.073 & $\mathrm{~S}$ & US & 0.08 \\
\hline 02 Dairy products and birds' eggs & $4.13^{* *}$ & $-0.223(4.586)^{* *}$ & 0.309 & $3.200 *$ & $\mathrm{~S}$ & $\mathrm{~S}$ & 0.27 \\
\hline 03 Fish, crustacean and molluscs, and preparations thereof & $7.20 * *$ & $-0.933(6.057)^{* *}$ & $5.774 * *$ & 0.311 & $\mathrm{~S}$ & $\mathrm{~S}$ & 0.71 \\
\hline 04 Cereals and cereal preparations & $8.69 * *$ & $-0.288(6.649) * *$ & 0.012 & 0.837 & $\mathrm{~S}$ & $\mathrm{~S}$ & 0.20 \\
\hline 05 Vegetables and fruit & $3.63^{*}$ & $-0.290(4.297)^{* *}$ & $4.98^{* *}$ & 2.61 & US & $\mathrm{S}$ & 0.59 \\
\hline 06 Sugar, sugar preparations, and honey & 2.67 & $-0.196(3.687) *$ & 0.466 & 0.144 & $\mathrm{~S}$ & $\mathrm{~S}$ & 0.36 \\
\hline 08 Feeding stuff for animals (not including unmilled cereals) & $8.43^{* *}$ & $-0.495(6.549)^{* *}$ & 0.086 & 1.448 & $\mathrm{~S}$ & US & 0.39 \\
\hline 09 Miscellaneous edible products and preparations & 2.90 & $-0.163(3.841) *$ & 0.314 & $10.159^{* *}$ & $\mathrm{~S}$ & $\mathrm{~S}$ & 0.35 \\
\hline 11 Beverages & 1.94 & $-0.115(3.146)$ & 0.179 & $2.721 *$ & $\mathrm{~S}$ & $\mathrm{~S}$ & 0.36 \\
\hline 21 Hides, skins and furskins, raw & 1.66 & $0.055(2.908)$ & 0.059 & 12.690 ** & $\mathrm{S}$ & $\mathrm{S}$ & 0.35 \\
\hline 22 Oil seeds and oleaginous fruit & $18.38^{* *}$ & $-1.312(9.673)^{* *}$ & 0.020 & $7.955 * *$ & $S$ & $S$ & 0.43 \\
\hline 23 Crude rubber (including synthetic and reclaimed) & $5.35 * *$ & $-0.358(5.216)^{* *}$ & 0.092 & 2.439 & $\mathrm{~S}$ & $\mathrm{~S}$ & 0.45 \\
\hline 24 Cork and wood & 1.93 & $-0.252(3.134)$ & 0.293 & 0.029 & $\mathrm{~S}$ & $\mathrm{~S}$ & 0.54 \\
\hline 25 Pulp and waste paper & $6.90^{* *}$ & $-0.324(5.928)^{* *}$ & 0.528 & 0.012 & $\mathrm{~S}$ & $\mathrm{~S}$ & 0.45 \\
\hline 26 Textile fibers (not wool tops) and their wastes (not in yarn) & $4.77^{* *}$ & $-0.379(4.929)^{* *}$ & 0.703 & 0.275 & $\mathrm{~S}$ & $\mathrm{~S}$ & 0.30 \\
\hline 27 Crude fertilizer and crude minerals & $11.44^{* *}$ & $-0.553(7.631)^{* *}$ & 1.619 & $8.018 * *$ & $\mathrm{~S}$ & $\mathrm{~S}$ & 0.33 \\
\hline 28 Metalliferous ores and metal scrap & $7.47^{* *}$ & $-0.377(6.168)^{* *}$ & 1.057 & 1.505 & $\mathrm{~S}$ & $\mathrm{~S}$ & 0.31 \\
\hline 33 Petroleum, petroleum products, and related materials & 0.32 & $-0.056(1.269)$ & 0.011 & $5.476 * *$ & $\mathrm{~S}$ & $\mathrm{~S}$ & 0.26 \\
\hline 41 Animal oils and fats & 1.06 & $-0.158(2.320)$ & 0.224 & 0.301 & $\mathrm{~S}$ & $\mathrm{~S}$ & 0.32 \\
\hline 42 Fixed vegetable oils and fats & $4.00^{*}$ & $-0.307(4.512)^{* *}$ & 0.816 & 0.072 & $\mathrm{~S}$ & $\mathrm{~S}$ & 0.44 \\
\hline 43 Animal and vegetable oils and fats, processed and waxes & $4.01 * *$ & $-0.388(4.516)^{* *}$ & 0.439 & $4.465^{* *}$ & $\mathrm{~S}$ & $\mathrm{~S}$ & 0.39 \\
\hline 51 Organic chemicals & $4.49 * *$ & $-0.247(4.779)^{* *}$ & 0.864 & 0.029 & $\mathrm{~S}$ & $\mathrm{~S}$ & 0.39 \\
\hline 52 Inorganic chemicals & 45.09 ** & $-0.945(15.141)^{* *}$ & 4.470 ** & 0.422 & S & US & 0.48 \\
\hline 53 Dyeing, tanning, and coloring materials & $8.29 * *$ & $-0.347(6.491)^{* *}$ & 0.194 & $2.916^{*}$ & $\mathrm{~S}$ & $\mathrm{~S}$ & 0.25 \\
\hline 54 Medicinal and pharmaceutical products & $5.59 * *$ & $-0.449(5.332)^{* *}$ & 1.932 & 0.142 & $\mathrm{~S}$ & $\mathrm{~S}$ & 0.48 \\
\hline 55 Oils and perfume materials; toilet and cleansing preparations & 3.11 & $-0.255(-3.981) *$ & 0.424 & 0.441 & $\mathrm{~S}$ & $\mathrm{~S}$ & 0.45 \\
\hline 56 Fertilizers, manufactured & $3.98^{*}$ & $-0.577(4.499)^{* *}$ & 0.292 & $10.609 * *$ & $\mathrm{~S}$ & $\mathrm{~S}$ & 0.53 \\
\hline 57 Explosives and pyrotechnic products & $11.28^{* *}$ & $-0.537(7.577)^{* *}$ & 1.658 & 0.073 & $\mathrm{~S}$ & $\mathrm{~S}$ & 0.41 \\
\hline 58 Artificial resins and plastic materials, and cellulose esters, etc & $13.90^{* *}$ & $-0.714(8.413)^{* *}$ & 0.389 & 2.359 & $\mathrm{~S}$ & $\mathrm{~S}$ & 0.39 \\
\hline 59 Chemical materials and products, nes & $10.69 * *$ & $-0.576(7.376)^{* *}$ & 1.759 & $4.636 * *$ & $\mathrm{~S}$ & $\mathrm{~S}$ & 0.46 \\
\hline 61 Leather, leather manufactures, nes, and dressed furskins & 1.19 & $-0.052(2.457)$ & 1.030 & 0.008 & $\mathrm{~S}$ & US & 0.25 \\
\hline 62 Rubber manufactures, nes & $3.81^{*}$ & $-0.158(4.401)^{* *}$ & $4.721 * *$ & $9.169 * *$ & $\mathrm{~S}$ & $\mathrm{~S}$ & 0.23 \\
\hline 63 Cork and wood, cork manufactures & $5.56^{* *}$ & $-0.289(5.322) * *$ & 1.375 & 1.095 & $\mathrm{~S}$ & $\mathrm{~S}$ & 0.28 \\
\hline 64 Paper, paperboard, and articles of pulp, of paper, or of paperboard & $4.43^{* *}$ & $-0.392(4.747)^{* *}$ & 4.193 ** & 1.557 & $\mathrm{~S}$ & $\mathrm{~S}$ & 0.34 \\
\hline 65 Textile yarn, fabrics, made-up articles, nes, and related products & 3.12 & $-0.175(3.986)^{*}$ & 0.488 & 0.257 & S & S & 0.31 \\
\hline
\end{tabular}


Table 4. Cont.

\begin{tabular}{|c|c|c|c|c|c|c|c|}
\hline \multirow[b]{2}{*}{ Industries } & \multicolumn{7}{|c|}{ Diagnostics } \\
\hline & F Stat ${ }^{a}$ & $\hat{\theta}_{0}(t \text {-Test })^{b}$ & $L M^{c}$ & $R E S E T^{d}$ & cUSUM & CUSUMSQ & $\operatorname{Adj} . R^{2}$ \\
\hline 66 Non-metallic mineral manufactures, nes & $10.37^{* *}$ & $-0.483(7.263)^{* *}$ & 0.091 & 0.486 & $\mathrm{~S}$ & $\mathrm{~S}$ & 0.35 \\
\hline 67 Iron and steel & $3.65^{*}$ & $-0.155(4.313)^{* *}$ & 0.034 & 0.011 & $\mathrm{~s}$ & $\mathrm{~S}$ & 0.41 \\
\hline 68 Non-ferrous metals. & $4.34^{* *}$ & $-0.349(4.699)^{* *}$ & $6.302 * *$ & 0.002 & $\mathrm{~S}$ & $\mathrm{~S}$ & 0.43 \\
\hline 69 Manufactures of metals, nes & $5.92 * *$ & $-0.386(5.489) * *$ & 0.000 & 1.777 & $\mathrm{~s}$ & $\mathrm{~S}$ & 0.41 \\
\hline 71 Power generating machinery and equipment & $9.32 * *$ & $-0.641(6.887)^{* *}$ & $2.796^{*}$ & 0.322 & $\mathrm{~S}$ & $\mathrm{~S}$ & 0.45 \\
\hline 72 Machinery specialized for particular industries & $5.61^{* *}$ & $-0.296(5.341) * *$ & 1.039 & 2.238 & $\mathrm{~s}$ & $\mathrm{~S}$ & 0.27 \\
\hline 73 Metalworking machinery & $3.91 *$ & $-0.237(4.460)^{* *}$ & 0.055 & $4.731 * *$ & $\mathrm{~S}$ & $\mathrm{~S}$ & 0.26 \\
\hline 74 General industrial machinery and equipment, nes, and parts of, nes & $3.53 *$ & $-0.281(4.235)^{* *}$ & 0.145 & 1.237 & $\mathrm{~S}$ & $\mathrm{~S}$ & 0.36 \\
\hline 75 Office machines and automatic data processing equipment & 2.69 & $-0.159(3.701) *$ & 0.881 & 1.255 & $\mathrm{~S}$ & $\mathrm{~S}$ & 0.48 \\
\hline 76 Telecommunications, sound recording, and reproducing equipment & $4.56^{* *}$ & $-0.327(4.824)^{* *}$ & 0.016 & $5.949 * *$ & $\mathrm{~s}$ & $\mathrm{~s}$ & 0.37 \\
\hline 77 Electric machinery, apparatus and appliances, nes, and parts, nes & $4.10^{* *}$ & $-0.212(4.568)^{* *}$ & 0.386 & 0.006 & $\mathrm{~S}$ & $\mathrm{~S}$ & 0.28 \\
\hline 78 Road vehicles & $8.00 * *$ & $-0.302(6.383) * *$ & 0.019 & 0.027 & $\mathrm{~s}$ & $\mathrm{~S}$ & 0.20 \\
\hline 79 Other transport equipment & $5.57^{* *}$ & $-0.534(5.326)^{* *}$ & 1.059 & 0.903 & $\mathrm{~S}$ & $\mathrm{~S}$ & 0.52 \\
\hline 81 Sanitary, plumbing, heating, lighting fixtures, and fittings, nes & 3.50 & $-0.287(4.223)^{* *}$ & 0.029 & 2.593 & $\mathrm{~S}$ & $\mathrm{~S}$ & 0.37 \\
\hline 82 Furniture and parts thereof & 2.48 & $-0.149(3.557)$ & 0.533 & 0.014 & $\mathrm{~s}$ & $\mathrm{~S}$ & 0.44 \\
\hline 83 Travel goods, handbags, and similar containers & 2.30 & $-0.223(3.423)$ & 1.088 & 0.005 & $\mathrm{~s}$ & $\mathrm{~S}$ & 0.47 \\
\hline 84 Articles of apparel and clothing accessories & $4.21 * *$ & $-0.355(4.637)^{* *}$ & 1.488 & $3.126^{*}$ & $\mathrm{~S}$ & $\mathrm{~S}$ & 0.47 \\
\hline 85 Footwear & 2.77 & $-0.048(3.752) *$ & $5.070 * *$ & 1.928 & $\mathrm{~S}$ & $\mathrm{~S}$ & 0.35 \\
\hline 87 Professional, scientific, controlling instruments, apparatus, nes & $5.06^{* *}$ & $-0.274(5.079) * *$ & 1.220 & 0.309 & $\mathrm{~s}$ & $\mathrm{~S}$ & 0.58 \\
\hline 89 Miscellaneous manufactured articles, nes & $4.20 * *$ & $-0.492(4.621) * *$ & 0.287 & $2.850 *$ & $\mathrm{~S}$ & $\mathrm{~S}$ & 0.47 \\
\hline 93 Special transactions, commodity not classified according to class & $5.35^{* *}$ & $-0.363(5.217)^{* *}$ & 1.012 & 0.053 & $\mathrm{~S}$ & $\mathrm{~S}$ & 0.40 \\
\hline 99 Estimate of non-Canadian low-value shipments & 2.76 & $-0.134(3.746) *$ & 1.847 & 0.015 & $\mathrm{~s}$ & $\mathrm{~S}$ & 0.27 \\
\hline
\end{tabular}

99 Estimate of non-Canadian low-value shipments

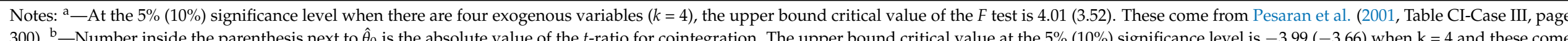

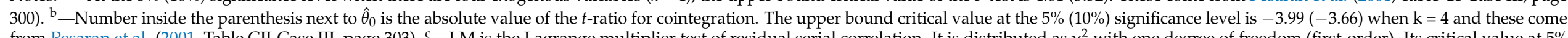

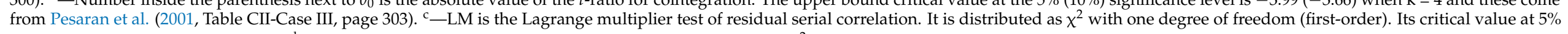
(10\%) significance level is 3.84 (2.77). d ${ }^{d}$-RESET is Ramsey's test for misspecification. It is distributed as $\chi^{2}$ with one degree of freedom. ${ }^{* *}$, and ${ }^{*}$ show a level of significance at $5 \%$ and $10 \%$, respectively. 
Due to the volume of the short-run estimates, we restrict ourselves to reporting short-run estimates attached to economic policy uncertainty variables. Those attached to $\triangle L n P U^{K O R}$ are reported in Table 1 and estimates attached to $\triangle L n P U^{U S}$ in Table 2. From Table 1 we gather that the Korean uncertainty measure carries at least one lagged significant coefficient in a total of 24 industries, implying that it has significant short-run effects. The comparable number for the U.S. policy uncertainty measure in Table 2 is 20 . Thus, it appears that the Korean policy uncertainty measure has relatively more significant shortrun effects on the U.S. exports to Korea than the U.S.'s own uncertainty measure. In how many industries do short-run effects translate into the long run? The answer is provided by Table 3 where we learn that the the Korean policy uncertainty measure carries a significant coefficient in 9 industries coded $05,25,29,51,58,67,69,79$, and $93 .{ }^{5}$ While in two industries (51 and 67) the coefficient estimate is positive, in the remaining seven industries it is negative. The aggregate trade share of these seven industries from Table 3 happens to be almost $9 \%$, implying that only $9 \%$ of the U.S. exports to Korea is hurt by increased policy uncertainty in Korea. As for the long-run effects of the U.S. policy uncertainty, it carries a significant coefficient in industries coded as $02,29,58,73,76$, and 87 . While in the first three industries the estimate is positive, in the last three industries it is negative. One of the large industries, i.e., 87 (professional, scientific, controlling instruments, apparatus, n.e.s. with $4.707 \%$ trade share) is in the last group. In this case, the aggregate share of the three industries that are hurt is almost $6 \%$. Therefore, even in the long run, an increase in Korean policy uncertainty is relatively more harmful to the U.S. exports to Korea, though the affected trade is small and marginal. ${ }^{6}$

Reported in Table 4 are a few additional diagnostic statistics. To make sure that the residuals in each model do not suffer from first-order serial correlation, we report the Lagrange multiplier test as $L M$, which is distributed as $\chi^{2}$ with one degree of freedom. As can be seen, it is insignificant in most industries, supporting autocorrelation-free residuals. The Ramsey RESET test is also reported as RESET and is used to identify misspecified models. This statistic is also distributed as $\chi^{2}$ with one degree of freedom. Again, since it is insignificant in most industries, the majority of optimum models are correctly specified. To establish stability of short-run and long-run coefficient estimates we apply the CUSUM and CUSUMSQ tests to the residuals of each model. Stable estimates are indicated by "S" and unstable ones by "US". As can be seen, there are hardly any unstable estimates. Finally, we report the size of adjusted $R^{2}$ to judge the goodness of the fit in each model.

Next, we consider estimates of the U.S. import demand model (4) or the Korean exports to the U.S. Again, results are reported in Tables 5-8. From Tables 5 and 6 we gather that the Korean economic policy uncertainty has significant short-run effects on exports of 26 Korean industries exporting to the U.S., whereas the U.S. policy uncertainty has significant effects only in 16 industries. Thus, it appears that Korea's own uncertainty measure has significant short-run effects in relatively more industries than the U.S. uncertainty measure. From Tables 7 and 8, however, we learn that short-run effects of the Korean policy uncertainty last into the long run only in industries 72 (machinery specialized for particular industries with $3.804 \%$ trade share), 78 (road vehicles with $27.34 \%$ trade share), and 88 (photographic equipment and supplies, optical goods, watches, etc. with $0.215 \%$ trade share). In the first two industries, the coefficient is positive, implying that an increase in Korean policy uncertainty boosts a total of $31.14 \%$ of Korean exports to the U.S. This could be the case if increased uncertainty in Korea makes U.S. importers more concerned for the future; hence, they import more today to avoid any future delay in delivery, production, and distribution. As for the U.S. policy uncertainty, it carries a significant coefficient in industries coded as $05,28,51,71,73$, and 87 . While in industry 28 (metalliferous ores and metal scrap with $0.021 \%$ trade share) the estimate is negative, in the reaming five industries it is positive. Since the aggregate trade share of these remaining five industries is $7.1 \%$, increased uncertainty in the U.S. will boost almost $7 \%$ of Korean exports to the U.S. ${ }^{7}$ 
Table 5. Short-run coefficient estimates of Korea economic policy uncertainty on U.S. imports from Korea.

\begin{tabular}{|c|c|c|c|c|c|c|c|c|}
\hline \multirow{2}{*}{ Code } & \multicolumn{8}{|c|}{ \# Lags on $\triangle P U^{K O R}$} \\
\hline & 0 & 1 & 2 & 3 & 4 & 5 & 6 & 7 \\
\hline 02 Dairy products and birds' eggs & 0.005 & $-0.365^{* *}$ & $-0.229^{* *}$ & $-0.225^{* *}$ & $-0.166^{*}$ & & & \\
\hline 03 Fish, crustacean and molluscs, and preparations thereof & 0.049 & $-0.146^{* *}$ & $-0.147^{* *}$ & & & & & \\
\hline 04 Cereals and cereal preparations & -0.033 & & & & & & & \\
\hline 05 Vegetables and fruit & 0.084 & $0.156^{* *}$ & & & & & & \\
\hline 06 Sugar, sugar preparations, and honey & -0.088 & & & & & & & \\
\hline 07 Coffee, tea, cocoa, spices, and manufactures thereof & 0.049 & & & & & & & \\
\hline 09 Miscellaneous edible products and preparations & -0.019 & & & & & & & \\
\hline 11 Beverages & 0.010 & $-0.077 *$ & $-0.136^{* *}$ & $-0.092^{* *}$ & $-0.156^{* *}$ & $-0.069 *$ & & \\
\hline 12 Tobacco and tobacco manufactures & -0.049 & & & & & & & \\
\hline 26 Textile fibers (not wool tops) and their wastes (not in yarn) & 0.059 & & & & & & & \\
\hline 27 Crude fertilizer and crude minerals & -0.005 & -0.23 & -0.092 & -0.059 & -0.081 & $0.152 * *$ & & \\
\hline 28 Metalliferous ores and metal scrap & 0.090 & & & & & & & \\
\hline 29 Crude animal and vegetable materials, nes & -0.026 & & & & & & & \\
\hline 33 Petroleum, petroleum products, and related materials & 0.008 & $-0.319 * *$ & $-0.316^{* *}$ & -0.091 & $-0.308^{* *}$ & & & \\
\hline 34 Gas, natural and manufactured & $-0.349^{* *}$ & & & & & & & \\
\hline 51 Organic chemicals & $-0.190^{* *}$ & & & & & & & \\
\hline 52 Inorganic chemicals & -0.113 & -0.086 & $-0.205^{* *}$ & & & & & \\
\hline 54 Medicinal and pharmaceutical products & 0.099 & 0.010 & 0.033 & -0.019 & $-0.454^{* *}$ & & & \\
\hline 55 Oils and perfume materials; toilet and cleansing preparations & -0.004 & & & & & & & \\
\hline 57 Explosives and pyrotechnic products & $-0.125^{* *}$ & $-0.090 * *$ & & & & & & \\
\hline 58 Artificial resins and plastic materials, and cellulose esters, etc & -0.031 & & & & & & & \\
\hline 59 Chemical materials and products, nes & -0.015 & $-0.143 * *$ & $-0.110^{* *}$ & $-0.085^{* *}$ & $-0.115^{* *}$ & $-0.112 * *$ & & \\
\hline 61 Leather, leather manufactures, nes, and dressed furskins & -0.028 & & & & & & & \\
\hline 62 Rubber manufactures, nes \# & 0.027 & & & & & & & \\
\hline 63 Cork and wood, cork manufactures & -0.082 & & & & & & & \\
\hline 64 Paper, paperboard, and articles of pulp, of paper, or of paperboard & $-0.044^{*}$ & & & & & & & \\
\hline 65 Textile yarn, fabrics, made-up articles, nes, and related products & $-0.032 *$ & $-0.051 * *$ & $-0.044^{* *}$ & $-0.047^{* *}$ & $-0.037^{* *}$ & $-0.039 * *$ & & \\
\hline 67 Iron and steel & $-0.171^{* *}$ & & & & & & & \\
\hline 68 Non-ferrous metals & 0.027 & & & & & & & \\
\hline 69 Manufactures of metals, nes & 0.006 & & & & & & & \\
\hline
\end{tabular}


Table 5. Cont

\begin{tabular}{|c|c|c|c|c|c|c|c|c|}
\hline \multirow{2}{*}{ Code } & \multicolumn{8}{|c|}{ \# Lags on $\triangle P U^{K O R}$} \\
\hline & 0 & 1 & 2 & 3 & 4 & 5 & 6 & 7 \\
\hline 71 Power generating machinery and equipment & $-0.152 * *$ & -0.057 & 0.018 & 0.018 & $-0.135^{* *}$ & & & \\
\hline 72 Machinery specialized for particular industries & 0.058 & $-0.155^{* *}$ & $-0.211^{* *}$ & $-0.087^{* *}$ & -0.059 & $-0.118^{* *}$ & & \\
\hline 73 Metalworking machinery & -0.065 & $-0.138^{* *}$ & & & & & & \\
\hline 74 General industrial machinery and equipment, nes, and parts of, nes & -0.038 & $-0.115^{* *}$ & -0.047 & $-0.065^{* *}$ & & & & \\
\hline 75 Office machines and automatic data processing equipment & -0.027 & & & & & & & \\
\hline 76 Telecommunications, sound recording, and reproducing equipment & -0.049 & & & & & & & \\
\hline 77 Electric machinery, apparatus and appliances, nes, and parts, nes & -0.033 & & & & & & & \\
\hline 78 Road vehicles & $0.148^{* *}$ & -0.021 & 0.030 & $-0.075^{* *}$ & $-0.141^{* *}$ & & & \\
\hline 79 Other transport equipment & -0.122 & -0.165 & $-0.263^{* *}$ & & & & & \\
\hline 81 Sanitary, plumbing, heating, lighting fixtures, and fittings, nes & 0.130 * & & & & & & & \\
\hline 82 Furniture and parts thereof & -0.008 & & & & & & & \\
\hline 83 Travel goods, handbags, and similar containers & -0.065 & & & & & & & \\
\hline 84 Articles of apparel and clothing accessories & -0.016 & 0.039 & $0.076^{*}$ & 0.021 & -0.042 & $-0.101^{* *}$ & $-0.064 * *$ & \\
\hline 85 Footwear & 0.014 & & & & & & & \\
\hline 87 Professional, scientific, controlling instruments, apparatus, nes & 0.009 & -0.060 & -0.042 & $0.104^{* *}$ & 0.039 & -0.029 & 0.048 & \\
\hline 88 Photographic equipment and supplies, optical goods, watches, etc & 0.032 & $0.095^{* *}$ & 0.027 & $0.094 * *$ & $0.086^{* *}$ & 0.035 & $0.088^{* *}$ & $0.139^{* *}$ \\
\hline 89 Miscellaneous manufactured articles, nes & -0.022 & $-0.061^{* *}$ & -0.014 & -0.034 & $-0.048^{* *}$ & -0.029 & $0.045^{* *}$ & \\
\hline 98 Estimate of import items valued under $\$ 251$ and others & 0.009 & & & & & & & \\
\hline
\end{tabular}

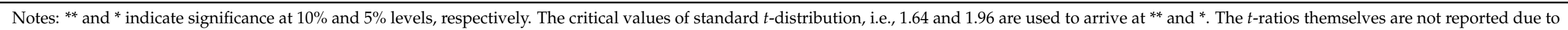
space constraints but they are available upon request. 
Table 6. Short-run coefficient estimates of U.S. economic policy uncertainty on U.S. imports from Korea.

\begin{tabular}{|c|c|c|c|c|c|c|c|c|}
\hline \multirow{2}{*}{ Code } & \multicolumn{8}{|c|}{ \# Lags on $\triangle P U^{U S}$} \\
\hline & 0 & 1 & 2 & 3 & 4 & 5 & 6 & 7 \\
\hline 02 Dairy products and birds' eggs & 0.112 & & & & & & & \\
\hline 03 Fish, crustacean and molluscs, and preparations thereof & 0.012 & & & & & & & \\
\hline 04 Cereals and cereal preparations & 0.006 & & & & & & & \\
\hline 05 Vegetables and fruit & $0.196^{* *}$ & $-0.279 * *$ & & & & & & \\
\hline 06 Sugar, sugar preparations, and honey & -0.091 & & & & & & & \\
\hline 07 Coffee, tea, cocoa, spices, and manufactures thereof & 0.113 & & & & & & & \\
\hline 09 Miscellaneous edible products and preparations & $0.066^{*}$ & & & & & & & \\
\hline 11 Beverages & 0.047 & & & & & & & \\
\hline 12 Tobacco and tobacco manufactures & 0.036 & & & & & & & \\
\hline 26 Textile fibers (not wool tops) and their wastes (not in yarn) & -0.068 & & & & & & & \\
\hline 27 Crude fertilizer and crude minerals & 0.089 & & & & & & & \\
\hline 28 Metalliferous ores and metal scrap & -0.101 & $0.580 * *$ & & & & & & \\
\hline 29 Crude animal and vegetable materials, nes & 0.016 & $-0.226^{* *}$ & & & & & & \\
\hline 33 Petroleum, petroleum products, and related materials & 0.278 & 0.254 & 0.238 & 0.165 & $0.715^{* *}$ & & & \\
\hline $34 \mathrm{Gas}$, natural and manufactured & 0.181 & & & & & & & \\
\hline 51 Organic chemicals & $0.214^{* *}$ & & & & & & & \\
\hline 52 Inorganic chemicals & 0.093 & 0.155 & $0.334^{* *}$ & $0.250 * *$ & 0.057 & -0.145 & $0.148^{*}$ & \\
\hline 54 Medicinal and pharmaceutical products & -0.089 & & & & & & & \\
\hline 55 Oils and perfume materials; toilet and cleansing preparations & 0.034 & & & & & & & \\
\hline 57 Explosives and pyrotechnic products & $0.129 * *$ & 0.073 * & & & & & & \\
\hline 58 Artificial resins and plastic materials, and cellulose esters, etc & 0.009 & & & & & & & \\
\hline 59 Chemical materials and products, nes & 0.086 & 0.097 & $0.128 * *$ & & & & & \\
\hline 61 Leather, leather manufactures, nes, and dressed furskins & -0.103 & & & & & & & \\
\hline 62 Rubber manufactures, nes \# & -0.020 & & & & & & & \\
\hline 63 Cork and wood, cork manufactures & 0.094 & & & & & & & \\
\hline 64 Paper, paperboard, and articles of pulp, of paper, or of paperboard & 0.035 & & & & & & & \\
\hline 65 Textile yarn, fabrics, made-up articles, nes, and related products & 0.012 & & & & & & & \\
\hline 67 Iron and steel & $0.236^{* *}$ & & & & & & & \\
\hline 68 Non-ferrous metals & -0.009 & & & & & & & \\
\hline 69 Manufactures of metals, nes & 0.029 & & & & & & & \\
\hline
\end{tabular}


Table 6. Cont

\begin{tabular}{|c|c|c|c|c|c|c|c|c|}
\hline \multirow{2}{*}{ Code } & \multicolumn{8}{|c|}{ \# Lags on $\triangle P U^{U S}$} \\
\hline & 0 & 1 & 2 & 3 & 4 & 5 & 6 & 7 \\
\hline 71 Power generating machinery and equipment & $0.132 * *$ & & & & & & & \\
\hline 72 Machinery specialized for particular industries & -0.040 & & & & & & & \\
\hline 73 Metalworking machinery & 0.117 & & & & & & & \\
\hline 74 General industrial machinery and equipment, nes, and parts of, nes & 0.036 & & & & & & & \\
\hline 75 Office machines and automatic data processing equipment & 0.001 & $-0.087^{* *}$ & & & & & & \\
\hline 76 Telecommunications, sound recording, and reproducing equipment & -0.036 & & & & & & & \\
\hline 77 Electric machinery, apparatus and appliances, nes, and parts, nes & 0.029 & & & & & & & \\
\hline 78 Road vehicles & $-0.141^{* *}$ & & & & & & & \\
\hline 79 Other transport equipment & 0.113 & & & & & & & \\
\hline 81 Sanitary, plumbing, heating, lighting fixtures, and fittings, nes & 0.144 * & & & & & & & \\
\hline 82 Furniture and parts thereof & 0.023 & & & & & & & \\
\hline 83 Travel goods, handbags, and similar containers & $0.148^{* *}$ & & & & & & & \\
\hline 84 Articles of apparel and clothing accessories & 0.056 & 0.045 & -0.052 & -0.056 & 0.068 & & & \\
\hline 85 Footwear & -0.062 & & & & & & & \\
\hline 87 Professional, scientific, controlling instruments, apparatus, nes & $0.082 *$ & -0.014 & $0.089 *$ & $-0.122 * *$ & & & & \\
\hline 88 Photographic equipment and supplies, optical goods, watches, etc & 0.019 & & & & & & & \\
\hline 98 Estimate of import items valued under $\$ 251$ and others & -0.012 & & & & & & & \\
\hline
\end{tabular}

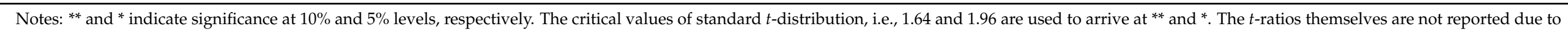
space constraints but they are available upon request. 
Table 7. Long-run coefficient estimates of U.S. import models.

\begin{tabular}{|c|c|c|c|c|c|c|}
\hline Industries & Share $^{\mathrm{c}}$ & Constant & $\operatorname{Ln} Y_{t}^{U S}$ & $\operatorname{Ln} R E X_{t}$ & $\operatorname{Ln} \mathrm{PU}_{t} \mathrm{KOR}^{\mathrm{KOR}}$ & ${ }^{L n} \mathrm{PU}_{t}{ }^{\mathrm{US}}$ \\
\hline 02 Dairy products and birds' eggs \# ${ }^{a}$ & $0.013 \%$ & $4.959(4.628)^{* *}$ & $3.828(2.095) * *$ & $-3.048(2.236)^{* *}$ & $0.325(1.012)^{b}$ & $0.305(1.015)$ \\
\hline 03 Fish, crustacean and molluscs, and preparations thereof \# & $0.207 \%$ & $2.381(2.672) * *$ & $1.673(0.987)$ & $-0.509(0.399)$ & $0.293(1.115)$ & $0.050(0.199)$ \\
\hline 04 Cereals and cereal preparations \# & $0.162 \%$ & $0.476(1.506)$ & $0.614(0.004)$ & $-17.538(0.056)$ & $-14.159(0.057)$ & $2.529(0.063)$ \\
\hline 05 Vegetables and fruit\# & $0.264 \%$ & $-7.136(5.353)^{* *}$ & $7.973(5.626)^{* *}$ & $-0.241(0.239)$ & $-0.156(0.625)$ & $1.105(3.706)^{* *}$ \\
\hline 06 Sugar, sugar preparations, and honey \# & $0.006 \%$ & $-4.945(6.838)^{* *}$ & $1.690(1.655) *$ & $2.135(2.948) * *$ & $-0.155(1.058)$ & $-0.160(0.959)$ \\
\hline 07 Coffee, tea, cocoa, spices, and manufactures thereof \# & $0.023 \%$ & $0.376(2.873)^{* *}$ & $4.197(1.573)$ & $-1.582(0.845)$ & $0.216(0.602)$ & $0.499(1.211)$ \\
\hline 09 Miscellaneous edible products and preparations\# & $0.276 \%$ & $-2.239(2.444)^{* *}$ & $3.579(0.605)$ & $-8.899(0.874)$ & $0.470(0.509)$ & $0.766(0.634)$ \\
\hline 11 Beverages & $0.147 \%$ & $1.498(2.245)^{* *}$ & $2.804(0.807)$ & $-2.679(1.086)$ & $0.338(0.607)$ & $0.538(1.018)$ \\
\hline 12 Tobacco and tobacco manufactures \# & $0.108 \%$ & $-8.897(4.623) * *$ & $8.999(3.972) * *$ & $2.459(1.528)$ & $-0.243(0.759)$ & $0.177(0.507)$ \\
\hline 27 Crude fertilizer and crude minerals \# & $0.025 \%$ & $-6.919(4.872)^{* *}$ & $5.109(3.492) * *$ & $1.237(1.248)$ & $-0.021(0.084)$ & $0.259(1.090)$ \\
\hline 28 Metalliferous ores and metal scrap \# & $0.021 \%$ & $26.479(14.544) * *$ & $0.468(0.238)$ & $-2.019(1.448)$ & $0.099(0.388)$ & $-0.744(2.329) * *$ \\
\hline 29 Crude animal and vegetable materials, nes\# & $0.052 \%$ & $6.505(5.944)^{* *}$ & $2.247(2.431)^{* *}$ & $-1.602(2.489)^{* *}$ & $0.205(1.377)$ & $0.137(0.781)$ \\
\hline 33 Petroleum, petroleum products, and related materials \# & $4.354 \%$ & $2.345(4.022) * *$ & $7.067(2.278)^{* *}$ & $-3.384(1.512)$ & $1.171(1.604)$ & $-1.149(1.119)$ \\
\hline $34 \mathrm{Gas}$, natural and manufactured & $0.021 \%$ & $-1.798(2.153)^{* *}$ & $5.596(0.876)$ & $-1.144(0.254)$ & $0.127(0.126)$ & $1.028(0.980)$ \\
\hline 51 Organic chemicals & $1.732 \%$ & $13.939(6.032) * *$ & $1.719(0.899)$ & $-4.796(3.524)^{* *}$ & $-0.040(0.158)$ & $0.629(2.27) * *$ \\
\hline 52 Inorganic chemicals \# & $0.159 \%$ & $-2.841(2.951) * *$ & $6.424(1.713) *$ & $0.587(0.224)$ & $0.272(0.448)$ & $-0.271(0.304)$ \\
\hline 54 Medicinal and pharmaceutical products & $2.833 \%$ & $-16.874(3.150) * *$ & $31.559(3.389) * *$ & $3.271(0.542)$ & $0.822(0.570)$ & $-0.816(0.562)$ \\
\hline 55 Oils and perfume materials; toilet and cleansing preparations \# & $0.828 \%$ & $3.521(2.639) * *$ & $16.423(2.108) * *$ & $5.391(0.922)$ & $2.408(1.337)$ & $-1.029(0.647)$ \\
\hline 57 Explosives and pyrotechnic products \# & $1.522 \%$ & $2.063(1.687) *$ & $85.955(0.117)$ & $17.065(0.096)$ & $2.092(0.102)$ & $-0.828(0.053)$ \\
\hline 58 Artificial resins and plastic materials, and cellulose esters, etc \# & $1.087 \%$ & $-4.139(2.914)^{* *}$ & $-8.386(0.529)$ & $-9.540(0.732)$ & $0.909(0.701)$ & $-0.255(0.294)$ \\
\hline 61 Leather, leather manufactures, nes, and dressed furskins & $0.006 \%$ & $11.891(3.479) * *$ & $-5.549(3.245) * *$ & $0.611(0.501)$ & $-0.088(0.360)$ & $-0.318(1.067)$ \\
\hline 62 Rubber manufactures, nes \# & $2.146 \%$ & $2.073(3.176)^{* *}$ & $-10.243(0.614)$ & $-0.453(0.090)$ & $0.903(0.719)$ & $-0.662(0.426)$ \\
\hline 63 Cork and wood, cork manufactures \# & $0.006 \%$ & $-6.161(3.089)^{* *}$ & $3.972(1.124)$ & $3.701(1.468)$ & $-0.429(0.875)$ & $0.489(0.769)$ \\
\hline 64 Paper, paperboard, and articles of pulp, of paper, or of paperboard \# & $0.849 \%$ & $1.342(4.451)^{* *}$ & $1.821(2.084)^{* *}$ & $0.371(0.615)$ & $0.073(0.529)$ & $-0.101(0.524)$ \\
\hline 65 Textile yarn, fabrics, made-up articles, nes, and related products & $1.231 \%$ & $0.734(2.810) * *$ & $-0.277(0.161)$ & $1.595(1.318)$ & $-0.358(1.242)$ & $0.149(0.517)$ \\
\hline 67 Iron and steel \# & $3.506 \%$ & $7.709(3.961)^{* *}$ & $0.319(0.114)$ & $-4.981(2.540)^{* *}$ & $0.193(0.474)$ & $0.514(1.106)$ \\
\hline 68 Non-ferrous metals \# & $1.095 \%$ & $7.664(3.845)^{* *}$ & $4.228(1.248)$ & $-7.151(2.812)^{* *}$ & $0.151(0.358)$ & $0.839(1.564)$ \\
\hline 69 Manufactures of metals, nes & $2.430 \%$ & $-0.625(3.623) * *$ & $3.864(7.068)^{* *}$ & $0.286(0.741)$ & $0.021(0.274)$ & $0.099(1.096)$ \\
\hline 71 Power generating machinery and equipment & $3.256 \%$ & $-1.337(3.786)^{* *}$ & $5.493(2.312) * *$ & $0.025(0.016)$ & $-0.555(1.439)$ & $0.866(2.372)^{* *}$ \\
\hline 72 Machinery specialized for particular industries & $3.804 \%$ & $10.219(5.099)^{* *}$ & $-4.756(1.177)$ & $-10.609(3.657) * *$ & $1.994(2.96)^{* *}$ & $-0.419(0.799)$ \\
\hline 73 Metalworking machinery & $0.832 \%$ & $11.321(5.779)^{* *}$ & $2.163(1.151)$ & $-5.239(3.718)^{* *}$ & $0.175(0.674)$ & $0.424(1.628) *$ \\
\hline 74 General industrial machinery and equipment, nes, and parts of, nes & $5.144 \%$ & $0.339(2.322) * *$ & $4.419(2.059) * *$ & $-1.059(0.703)$ & $0.265(0.812)$ & $0.325(1.029)$ \\
\hline 75 Office machines and automatic data processing equipment & $6.193 \%$ & $-1.250(3.599)^{* *}$ & $1.311(0.546)$ & $3.769(2.245) * *$ & $-0.268(0.801)$ & $0.228(0.513)$ \\
\hline 76 Telecommunications, sound recording, and reproducing equipment & $5.641 \%$ & $10.912(5.509)^{* *}$ & $-3.595(3.151) * *$ & $-0.103(0.130)$ & $-0.178(1.071)$ & $-0.128(0.691)$ \\
\hline 77 Electric machinery, apparatus and appliances, nes, and parts, nes \# & $11.154 \%$ & $1.103(5.232) * *$ & $1.807(2.937) * *$ & $0.987(2.263) * *$ & $-0.143(1.571)$ & $0.130(1.239)$ \\
\hline 78 Road vehicles \# & $27.347 \%$ & $-0.411(4.904)^{* *}$ & $4.388(4.925)^{* *}$ & $0.248(0.395)$ & $0.359(2.173) * *$ & $-0.283(1.557)$ \\
\hline 79 Other transport equipment & $1.093 \%$ & $6.521(3.919) * *$ & $2.965(1.133)$ & $-2.528(1.399)$ & $0.364(0.864)$ & $0.313(0.764)$ \\
\hline
\end{tabular}


Table 7. Cont.

\begin{tabular}{|c|c|c|c|c|c|c|}
\hline Industries & Share $^{\mathrm{c}}$ & Constant & $\operatorname{Ln} Y_{t}^{U S}$ & Ln REX & $\operatorname{Ln} \mathrm{PU}_{t}{ }^{\mathrm{KOR}}$ & $\operatorname{Ln} P U_{t}{ }^{U S}$ \\
\hline 81 Sanitary, plumbing, heating, lighting fixtures, and fittings, nes & $0.183 \%$ & $-11.502(3.688)^{* *}$ & $11.859(4.000) * *$ & $3.493(1.744) *$ & $-0.596(1.264)$ & $0.821(1.578)$ \\
\hline 82 Furniture and parts thereof & $0.329 \%$ & $1.424(1.331)$ & $0.001(0.001)$ & $-2.336(0.583)$ & $-0.173(0.239)$ & $0.513(0.616)$ \\
\hline 83 Travel goods, handbags, and similar containers & $0.106 \%$ & $-5.251(3.508) * *$ & $10.232(2.100)^{* *}$ & $2.907(0.742)$ & $-0.714(1.001)$ & $1.616(1.518)$ \\
\hline 84 Articles of apparel and clothing accessories & $0.320 \%$ & $-2.256(2.329) * *$ & $4.049(0.334)$ & $14.958(1.547)$ & $-1.954(0.882)$ & $0.789(0.268)$ \\
\hline 85 Footwear & $0.074 \%$ & $-5.997(4.117)^{* *}$ & $9.519(1.614) *$ & $5.621(1.581)$ & $1.390(1.182)$ & $-0.744(0.856)$ \\
\hline 87 Professional, scientific, controlling instruments, apparatus, nes & $1.022 \%$ & $3.837(4.242) * *$ & $2.336(1.139)$ & $-4.459(2.810) * *$ & $-0.462(1.001)$ & $1.252(2.572) * *$ \\
\hline 88 Photographic equipment and supplies, optical goods; watches, etc \# & $0.215 \%$ & $0.866(3.806) * *$ & $1.261(1.194)$ & $1.164(1.573)$ & $-0.340(1.827)$ * & $0.079(0.478)$ \\
\hline 89 Miscellaneous manufactured articles, nes & $4.262 \%$ & $-3.116(3.697) * *$ & $10.327(2.502)^{* * *}$ & $1.889(1.091)$ & $0.316(0.654)$ & $0.529(1.118)$ \\
\hline 93 Special transactions, commodity not classified according to class \# & $1.476 \%$ & $-13.649(9.581)^{* *}$ & $4.394(7.344)^{* *}$ & $1.869(4.444) * *$ & $0.005(0.079)$ & $-0.124(1.544)$ \\
\hline 98 Estimate of import items valued under $\$ 251$ and others \# & $0.454 \%$ & $3.025(4.319) * *$ & $0.906(1.133)$ & $-0.210(0.393)$ & $0.041(0.390)$ & $-0.058(0.465)$ \\
\hline
\end{tabular}

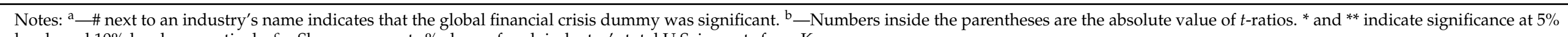

levels and $10 \%$ levels respectively. ${ }^{c}$-Share represents $\%$ share of each industry's total U.S. imports from Korea.

Table 8. Diagnostic statistics associated with U.S. import models.

\begin{tabular}{|c|c|c|c|c|c|c|c|}
\hline \multirow[b]{2}{*}{ Industries } & \multicolumn{7}{|c|}{ Diagnostics } \\
\hline & F Stat ${ }^{a}$ & $\hat{\rho}_{0}(t \text {-Test })^{b}$ & $L M^{c}$ & $R E S E T^{d}$ & сUSUм & cUSUMSQ & $\operatorname{Adj} . R^{2}$ \\
\hline 02 Dairy products and birds' eggs & $4.13^{* *}$ & $-0.367(4.585)^{* *}$ & 2.570 & 0.015 & US & $\mathrm{S}$ & 0.42 \\
\hline 03 Fish, crustacean and molluscs, and preparations thereof & 1.39 & $-0.234(2.662)$ & 1.340 & 0.012 & $\mathrm{~S}$ & $\mathrm{~S}$ & 0.43 \\
\hline 04 Cereals and cereal preparations & 0.39 & $-0.002(1.409)$ & $3.092 *$ & 1.188 & $\mathrm{~S}$ & $\mathrm{~S}$ & 0.39 \\
\hline 05 Vegetables and fruit & $5.66^{* *}$ & $-0.293(5.370)^{* *}$ & $22.259 * *$ & 1.188 & US & $\mathrm{S}$ & 0.45 \\
\hline 06 Sugar, sugar preparations, and honey & $9.19^{* *}$ & $-0.570(6.839) * *$ & 1.974 & 0.221 & $\mathrm{~S}$ & $\mathrm{~S}$ & 0.44 \\
\hline 07 Coffee, tea, cocoa, spices, and manufactures thereof & 1.57 & $-0.226(2.827)$ & 0.524 & 0.143 & $\mathrm{~S}$ & $\mathrm{~S}$ & 0.42 \\
\hline 09 Miscellaneous edible products and preparations & 1.22 & $0.041(2.493)$ & $4.530 * *$ & 0.325 & $\mathrm{~S}$ & $\mathrm{~S}$ & 0.40 \\
\hline 11 Beverages & 0.95 & $-0.087(2.195)$ & 0.362 & 1.315 & $\mathrm{~S}$ & $\mathrm{~S}$ & 0.32 \\
\hline 12 Tobacco and tobacco manufactures & $4.25^{* *}$ & $-0.204(4.649)^{* *}$ & 0.013 & 0.136 & $\mathrm{~S}$ & $\mathrm{~S}$ & 0.41 \\
\hline 26 Textile fibers (not wool tops) and their wastes (not in yarn) & $17.51^{* *}$ & $-0.553(9.441)^{* *}$ & 0.332 & 0.936 & $\mathrm{~S}$ & $\mathrm{~S}$ & 0.37 \\
\hline 27 Crude fertilizer and crude minerals & $4.67^{* *}$ & $-0.346(4.873)^{* *}$ & 0.313 & 0.009 & $\mathrm{~s}$ & $\mathrm{~S}$ & 0.42 \\
\hline 28 Metalliferous ores and metal scrap & $41.66^{* *}$ & $-0.917(14.558)^{* *}$ & 0.003 & 0.123 & $\mathrm{~S}$ & US & 0.46 \\
\hline 29 Crude animal and vegetable materials, nes & $6.89^{* *}$ & $-0.469(5.922) * *$ & 2.139 & 0.926 & $\mathrm{~S}$ & $\mathrm{~S}$ & 0.46 \\
\hline 33 Petroleum, petroleum products, and related materials & 3.06 & $-0.249(3.950) *$ & 0.635 & $4.598 * *$ & $\mathrm{~S}$ & $\mathrm{~S}$ & 0.46 \\
\hline 34 Gas, natural and manufactured & 0.94 & $-0.176(2.184)$ & 0.466 & 2.605 & $\mathrm{~S}$ & US & 0.38 \\
\hline
\end{tabular}


Table 8. Cont.

\begin{tabular}{|c|c|c|c|c|c|c|c|}
\hline \multirow[b]{2}{*}{ Industries } & \multicolumn{7}{|c|}{ Diagnostics } \\
\hline & F Stat ${ }^{a}$ & $\hat{\rho}_{0}(t \text {-Test })^{b}$ & $L M^{c}$ & $R E S E T^{d}$ & cusuM & cusumsQ & $\operatorname{Adj} . R^{2}$ \\
\hline 51 Organic chemicals & $7.13 * *$ & $-0.340(6.024)^{* *}$ & 0.048 & 2.158 & $\mathrm{~S}$ & $\mathrm{~S}$ & 0.27 \\
\hline 52 Inorganic chemicals & 1.72 & $-0.154(2.963)$ & 1.876 & 2.436 & $\mathrm{~S}$ & $\mathrm{~S}$ & 0.38 \\
\hline 55 Oils and perfume materials; toilet and cleansing preparations & 1.32 & $0.033(2.599)$ & 1.103 & 0.786 & US & $\mathrm{S}$ & 0.41 \\
\hline 57 Explosives and pyrotechnic products & 0.55 & $0.004(1.672)$ & 1.888 & 0.096 & $\mathrm{~S}$ & $\mathrm{~S}$ & 0.33 \\
\hline 58 Artificial resins and plastic materials, and cellulose esters, etc & 1.68 & $0.034(2.927)$ & $8.993 * *$ & 0.859 & $\mathrm{~S}$ & $\mathrm{~S}$ & 0.37 \\
\hline 59 Chemical materials and products, nes & 1.41 & $-0.136(2.684)$ & 0.030 & $14.499 * *$ & $\mathrm{~s}$ & $\mathrm{~S}$ & 0.33 \\
\hline 61 Leather, leather manufactures, nes, and dressed furskins & 2.39 & $-0.324(3.489)$ & 0.642 & $5.063 * *$ & $\mathrm{~S}$ & $\mathrm{~S}$ & 0.47 \\
\hline 63 Cork and wood, cork manufactures & 1.88 & $-0.192(3.091)$ & 0.088 & 2.057 & $\mathrm{~S}$ & $\mathrm{~S}$ & 0.37 \\
\hline 64 Paper, paperboard, and articles of pulp, of paper, or of paperboard & 3.90 * & $-0.209(4.454)^{* *}$ & 0.356 & 0.089 & $\mathrm{~S}$ & $\mathrm{~S}$ & 0.38 \\
\hline 65 Textile yarn, fabrics, made-up articles, nes, and related products & 1.53 & $-0.079(2.792)$ & 2.831 * & 0.279 & $\mathrm{~S}$ & $\mathrm{~S}$ & 0.46 \\
\hline 67 Iron and steel & 3.08 & $-0.157(3.962) *$ & 0.397 & 0.200 & $\mathrm{~s}$ & $\mathrm{~S}$ & 0.41 \\
\hline 68 Non-ferrous metals & 2.89 & $-0.177(3.834) *$ & 0.056 & 0.005 & $\mathrm{~s}$ & $\mathrm{~S}$ & 0.37 \\
\hline 69 Manufactures of metals, nes & 2.62 & $-0.293(3.651)$ & 0.003 & 1.667 & $\mathrm{~S}$ & $\mathrm{~S}$ & 0.49 \\
\hline 71 Power generating machinery and equipment & 2.90 & $-0.153(3.841) *$ & 0.029 & 2.541 & $\mathrm{~S}$ & $\mathrm{~S}$ & 0.44 \\
\hline 72 Machinery specialized for particular industries & $5.07 * *$ & $-0.094(5.083)^{* *}$ & 0.000 & $9.702 * *$ & $\mathrm{~S}$ & $\mathrm{~S}$ & 0.39 \\
\hline 73 Metalworking machinery & $6.54 * *$ & $-0.275(5.771)^{* *}$ & 1.246 & 0.895 & $\mathrm{~S}$ & $\mathrm{~S}$ & 0.31 \\
\hline 74 General industrial machinery and equipment, nes, and parts of, nes & 1.02 & $-0.111(2.279)$ & 0.645 & 0.026 & US & $\mathrm{S}$ & 0.27 \\
\hline 77 Electric machinery, apparatus and appliances, nes, and parts, nes & $5.40 * *$ & $-0.229(5.245)^{* *}$ & 0.129 & 3.551 * & $\mathrm{S}$ & $\mathrm{S}$ & 0.31 \\
\hline 78 Road vehicles & $5.24 * *$ & $-0.242(5.165)^{* *}$ & 0.256 & 0.222 & $\mathrm{~S}$ & $\mathrm{~S}$ & 0.35 \\
\hline 79 Other transport equipment & 3.01 & $-0.360(3.916) *$ & 2.526 & 0.808 & US & $\mathrm{S}$ & 0.45 \\
\hline 81 Sanitary, plumbing, heating, lighting fixtures, and fittings, nes & 2.68 & $-0.176(3.696) *$ & $3.024 *$ & 1.387 & $\mathrm{~S}$ & $\mathrm{~S}$ & 0.31 \\
\hline 82 Furniture and parts thereof & 0.34 & $-0.045(1.319)$ & 0.024 & 0.007 & US & $\mathrm{S}$ & 0.40 \\
\hline 83 Travel goods, handbags, and similar containers & 2.41 & $-0.092(3.503)$ & $5.433^{* *}$ & 0.209 & $\mathrm{~S}$ & $\mathrm{~S}$ & 0.26 \\
\hline 84 Articles of apparel and clothing accessories & 1.04 & $-0.022(2.305)$ & $7.209 * *$ & $3.453 *$ & $\mathrm{~s}$ & $\mathrm{~S}$ & 0.44 \\
\hline 85 Footwear & 3.31 & $-0.083(4.104) * *$ & 1.363 & 0.337 & $\mathrm{~S}$ & $\mathrm{~S}$ & 0.39 \\
\hline 87 Professional, scientific, controlling instruments, apparatus, nes & $3.52 *$ & $-0.112(4.237)^{* *}$ & 2.336 & 0.448 & $\mathrm{~S}$ & $\mathrm{~S}$ & 0.48 \\
\hline 88 Photographic equipment and supplies, optical goods, watches, etc & 2.91 & $-0.246(3.853) *$ & 0.006 & 2.376 & $\mathrm{~S}$ & $\mathrm{~S}$ & 0.44 \\
\hline 89 Miscellaneous manufactured articles, nes & 2.71 & $-0.067(3.716) *$ & 1.012 & $15.944 * *$ & $\mathrm{~S}$ & $\mathrm{~S}$ & 0.28 \\
\hline 93 Special transactions, commodity not classified according to class & $18.06 * *$ & $-0.903(9.589)^{* *}$ & 0.014 & 0.647 & $\mathrm{~S}$ & $\mathrm{~S}$ & 0.54 \\
\hline 98 Estimate of import items valued under $\$ 251$ and others & $3.66 *$ & $-0.212(4.313) * *$ & 0.004 & 0.004 & $\mathrm{~s}$ & $\mathrm{~S}$ & 0.27 \\
\hline
\end{tabular}

98 Estimate of import items valued under $\$ 251$ and others

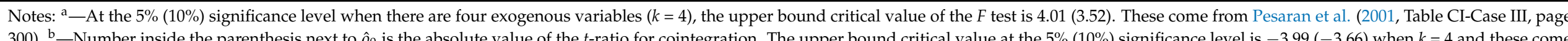

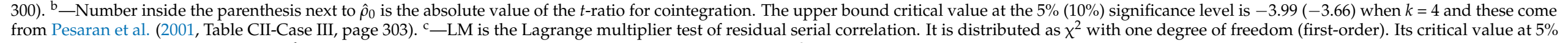
$(10 \%)$ significance level is 3.84 (2.77). d-RESET is Ramsey's test for misspecification. It is distributed as $\chi^{2}$ with one degree of freedom. ${ }^{* *}$, and ${ }^{*}$ show a level of significance at $5 \%$ and $10 \%$, respectively. 


\section{Summary and Conclusions}

The most comprehensive measure of uncertainty in every country is said to be the new policy uncertainty measure introduced by Baker et al. (2016). This news-based measure captures all uncertainties associated with economic or political factors, and since its introduction, researchers have tried to assess its impact on different macro variables such as stock returns, oil prices, domestic investment, capital flows, demand for money, the housing market, etc.

In this paper, we consider the trade flows between Korea and the U.S. and try to find out whose policy uncertainty measure has relatively more effects on each country's exports to the other. Depending on the availability of the data, we include 61 2-digit U.S. exporting industries to Korea and 49 2-digit Korean exporting industries to the U.S. Monthly data over the period 2000M1-2020M7 are used to carry out the empirical analysis. Our results could be best summarized by saying that both uncertainty measures had significant shortrun negative effects on 1/3rd of the industries exporting from the U.S. to Korea and from Korea to the U.S. However, short-run effects lasted into the long run in a limited number of industries. More precisely, Korean policy uncertainty had significantly negative long-run effects on 9 U.S. exporting industries which engaged in $9 \%$ of exports whereas, the U.S. policy uncertainty had negative effects in five industries which engaged in only $6 \%$ of exports. As for the effects of both uncertainty measure on the U.S. imports from Korea or Korean exports to the U.S., we found that again, both measures had significant short-run effects in almost one-third of the industries. Short-run effects were translated into the long run in three industries as far as the effects of the Korean uncertainty is concerned and in six industries as far as the U.S. policy uncertainty measure is concerned. An interesting finding is that both uncertainty measures had positive effects on Korean exports to the U.S., and while the total share of industries affected by the Korean uncertainty measure was $31 \%$, that of the industries affected by the U.S. uncertainty measure was $7 \%$. Thus, increase uncertainty in Korea induces the U.S. importers to import more. This could be the case if the U.S. importers expect political and economic situations in Korea to get worse as a result of increased uncertainty. For example, a small threat by North Korea will make the South Korean policy uncertainty measure rise. If this is perceived by the U.S. importers as a serious long-lasting threat, they will import more in order not to experience disturbance in their sales, delivery, and production. It should be noted that in the exchange rate uncertainty trade literature, the new direction is to assess the possibility of an asymmetric response of trade flows to a measure of exchange rate uncertainty advanced by Bahmani-Oskooee and Aftab (2017). Future research should investigate the possibility of asymmetric effects of policy uncertainty on trade flows.

Author Contributions: Both authors have contributed equally. All authors have read and agreed to the published version of the manuscript.

Funding: This research received no external funding.

Acknowledgments: Valuable comments of three anonymous reviewers are greatly appreciated. Remaining errors, however, are our own.

Conflicts of Interest: The authors declare no conflict of interest.

\section{Appendix A. Data Definitions and Sources}

Monthly data over 2000M1-2020M7 are used to carry out the empirical analysis. The data sources are as follows:
a. U.S. International Trade Commission (USITC).
b. International Financial Statistics of the IMF (www.imfstatistics.org). (accessed on 15 February 2021)
c. Economic Policy Uncertainty Group: https://www.policyuncertainty.com/. (accessed on 15 February 2021) 


\section{Variables}

$\boldsymbol{X}_{\mathbf{i}}{ }^{\text {US }}=$ Volume of exports of commodity $i$ by the U.S. to Korea. Export value data in U.S. dollars for each industry come from source a. In the absence of export prices at the commodity level, we follow Bahmani-Oskooee and Hegerty (2009) and use the aggregate export price index of the U.S. to deflate the nominal exports of each commodity. The aggregate export price index comes from source b.

$\boldsymbol{M}_{\mathbf{i}}{ }^{\text {US }}=$ Volume of imports of commodity $i$ by the U.S. from Korea. Import value data in U.S. dollars for each industry come from source a. Again, we use the aggregate import price index of the U.S. to deflate the nominal imports of each commodity. The aggregate import price index comes from source $b$.

$Y^{\mathrm{US}}=$ Measure of U.S. economic activity. Since data are monthly, we follow Bahmani-Oskooee and Ardalani (2006) and Bahmani-Oskooee and Aftab (2017) and use the Industrial Production Index, which is available monthly from source $b$.

$Y^{\mathrm{KOR}}=$ Measure of Korea's economic activity, also proxied by the industrial production index from source $b$.

$\boldsymbol{R E X}=$ Real bilateral exchange rate between US dollar and Korean won. It is defined as $\left(\mathrm{NEX}^{*} \mathrm{CPI}{ }^{\mathrm{US}}\right) / \mathrm{CPI}^{\mathrm{KOR}}$ where $N E X_{i}$ is the nominal exchange rate defined as the number of won per US dollar, CPI ${ }^{\mathrm{US}}$ is the price level in the United States and $\mathrm{CPI}^{\mathrm{KOR}}$ is the price level in Korea. Thus, a decline in REX reflects a real depreciation of the USD. All data come from Source b.

$P \boldsymbol{U}^{\mathrm{US}}$ = Measure of Economic Policy Uncertainty in the U.S., proxied by the economic policy uncertainty index from source c.

$P \boldsymbol{U}^{\mathrm{KO}} \underline{\mathrm{R}}$ Measure of Economic Policy Uncertainty in Korea, also proxied by the economic policy uncertainty index from source c.

\section{Notes}

1 The policy uncertainty measure is now constructed for more than 20 countries by the Policy Uncertainty Group. For more details visit www.policyuncertainty.com (accessed on 4 March 2021).

2 A few examples are Bahmani-Oskooee (1986), Bahmani-Oskooee and Niroomand (1998), and Bahmani-Oskooee and Kara (2005). Bahmani-Oskooee et al. (2013) is the latest review article.

3 Note that estimates of $\theta_{0}$ and $\rho_{0}$ in this context are the same as an estimate of the coefficient attached to the lagged error-correction term in Engle and Granger's (1987) approach. Hence, these estimates must be negative, and they measure the speed of adjustment. For proof, see Banerjee et al. (1998) and Bahmani-Oskooee and Ghodsi (2018).

4 For some other applications of this approach, see Halicioglu (2007), Durmaz (2015), Al-Shayeb and Hatemi-J (2016), Aftab et al. (2017), Arize et al. (2017), and Hajilee and Niroomand (2019).

5 By meaningful we mean cointegration is supported either by the $F$ or the $t$-test that is reported in the diagnostics in Table 4 . Note that in industry 82 , although the Korean policy uncertainty measure carries a significant coefficient, this industry is not included in the list since neither the F nor the $t$-test is significant.

6 As for the long-run effects of the income and exchange rate, while the exchange rate is significant in limited number of industries, Korean income or economic activity is significant in most industries.

7 Other diagnostics in Table 7 are similar to those in Table 4 and need no repeat. Furthermore, in most models the level of economic activity in the U.S. seems to be a significant long-run determinant of the U.S. imports from Korea.

\section{References}

Aftab, Muhammad, Karim Bux Shah Syed, and Naveed Katper. 2017. Exchange-rate Volatility and Malaysian-Thai Bilateral Industry Trade Flows. Journal of Economic Studies 44: 99-114. [CrossRef]

Al-Shayeb, Abdulrahman, and Abdulnasser Hatemi-J. 2016. Trade Openness and Economic Development in the UAE: An Asymmetric Approach. Journal of Economic Studies 43: 587-97. [CrossRef]

Al-Thaqeb, Saud Asaad, and Barrak Ghanim Algharabali. 2019. Economic Policy Uncertainty: A Literature Review. Journal of Economic Asymmetries 20: e00133. [CrossRef]

Arize, Augustine C., John Malindretos, and Emmanuel U. Igwe. 2017. Do Exchange Rate Changes Improve the Trade Balance: An Asymmetric Nonlinear Cointegration Approach. International Review of Economics and Finance 49: 313-26. [CrossRef]

Armelius, Hanna, Carl-Johan Belfrage, and Hanna Stenbacka. 2014. The Mystery of the Missing World Trade Growth after the Global Financial Crisis. Sveriges Riksbank Economic Review 3: 7-22. 
Bahmani-Oskooee, Mohsen. 1986. Determinants of International Trade Flows: Case of Developing Countries. Journal of Development Economics 20: 107-23. [CrossRef]

Bahmani-Oskooee, Mohsen, and Farhang Niroomand. 1998. Long-Run Price Elasticities and the Marshall-Lerner Condition Revisited. Economics Letters 61: 101-9. [CrossRef]

Bahmani-Oskooee, Mohsen, and Orhan Kara. 2005. Income and Price Elasticities of Trade: Some New Estimates. International Trade Journal 19: 165-78. [CrossRef]

Bahmani-Oskooee, Mohsen, and Zohre Ardalani. 2006. Exchange Rate Sensitivity of U.S. Trade Flows: Evidence from Industry Data. Southern Economic Journal 72: 542-59. [CrossRef]

Bahmani-Oskooee, Mohsen, and Scott W. Hegerty. 2007. Exchange Rate Volatility and Trade Flows: A Review Article. Journal of Economic Studies 34: 211-55. [CrossRef]

Bahmani-Oskooee, Mohsen, and Scott W. Hegerty. 2009. The Effects of Exchange-Rate Volatility on Commodity Trade between the U.S. and Mexico. Southern Economic Journal 75: 1019-44. [CrossRef]

Bahmani-Oskooee, Mohsen, and Muhammad Aftab. 2017. On the Asymmetric Effects of Exchange Rate Volatility on Trade Flows: New Evidence from US-Malaysia Trade at Industry Level. Economic Modelling 63: 86-103. [CrossRef]

Bahmani-Oskooee, Mohsen, and Seyed Hesam Ghodsi. 2017. Policy Uncertainty and House Prices in the United States of America. Journal of Real Estate Portfolio Management 23: 73-85. [CrossRef]

Bahmani-Oskooee, Mohsen, and Seyed Hesam Ghodsi. 2018. Asymmetric Causality between the U.S. Housing Market and its Stock Market: Evidence from State-Level Data. Journal of Economic Asymmetries 18: e00095. [CrossRef]

Bahmani-Oskooee, Mohsen, Hanafiah Harvey, and Scott W. Hegerty. 2013. Empirical Tests of the Marshall-Lerner Condition: A Literature Review. Journal of Economic Studies 40: 411-43. [CrossRef]

Bahmani-Oskooee, Mohsen, Ali Kutan, and Alice Kones. 2016. Policy Uncertainty and the Demand for Money in the United States. Applied Economics Quarterly 62: 37-49. [CrossRef]

Bahmani-Oskooee, Mohsen, and Mehrnoosh Hasanzade. 2021. Policy Uncertainty and Income Distribution: Asymmetric Evidence from State-Level Data in the U.S. Bulletin of Economic Research. forthcoming. [CrossRef]

Bahmani-Oskooee, Mohsen, Hanafiah Harvey, and Farhang Niroomand. 2018. On the Impact of Policy Uncertainty on Oil Prices: An Asymmetry Analysis. International Journal of Financial Studies 6: 12. [CrossRef]

Baker, Scott R., Nicholas Bloom, and Steven J. Davis. 2016. Measuring Economic Policy Uncertainty. Quarterly Journal of Economics 131: 1593-636. [CrossRef]

Banerjee, Anindya, Juan Dolado, and Ricardo Mestre. 1998. Error-Correction Mechanism Tests in a Single Equation Framework. Journal of Time Series Analysis 19: 267-85. [CrossRef]

Brogaard, Jonathan, and Andrew Detzel. 2015. The Asset-Pricing Implications of Government Economic Policy Uncertainty. Management Science 61: 3-18. [CrossRef]

Durmaz, Nazif. 2015. Industry Level J-curve in Turkey. Journal of Economic Studies 42: 689-706. [CrossRef]

Engle, Robert F., and C. W. J. Granger. 1987. Cointegration and Error Correction: Representation, Estimation, and Testing. Econometrica 55: 251-76. [CrossRef]

Hajilee, Massomeh, and Farhang Niroomand. 2019. On the Link between Financial Market Inclusion and Trade Openness: An Asymmetric Analysis. Economic Analysis and Policy 62: 373-81. [CrossRef]

Halicioglu, Ferda. 2007. The J-Curve Dynamics of Turkish Bilateral Trade: A Cointegration Approach. Journal of Economic Studies 34: 103-19. [CrossRef]

Han, Liyan, Mengchao Qi, and Libo Yin. 2016. Macroeconomic Policy Uncertainty Shocks on the Chinese Economy: A GVAR Analysis. Applied Economics 48: 4907-21. [CrossRef]

Istiak, Khandokar, and Md Rafayet Alam. 2019. Oil Prices, Policy Uncertainty, and Asymmetries in Inflation Expectations. Journal of Economic Studies 46: 324-34. [CrossRef]

Kang, Wensheng, and Ronald A. Ratti. 2013. Oil Shocks, Policy Uncertainty, and Stock Market Return. International Financial Markets, Institutions, and Money 26: 305-18. [CrossRef]

Ko, Jun-Hyung, and Chang-Min Lee. 2015. International Economic Policy Uncertainty and Stock Prices: Wavelet Approach. Economics Letters 134: 118-22. [CrossRef]

Pastor, L'uboš, and Pietro Veronesi. 2013. Policy Uncertainty and Risk Premia. Journal of Financial Economics 110: 520-45. [CrossRef]

Pesaran, Hashem M., Yongcheol Shin, and Richard J. Smith. 2001. Bounds Testing Approach to the Analysis of Level Relationships. Journal of Applied Econometrics 16: 289-326. [CrossRef]

Tam, Pui Sun. 2018. Global trade flows and economic policy uncertainty. Applied Economics 50: 3718-34. [CrossRef]

Wang, Yizhong, Carl R. Chen, and Ying Sophie Huang. 2014. Economic Policy Uncertainty and Corporate Investment: Evidence from China. Pacific-Basin Finance Journal 26: 227-43. [CrossRef] 